(c) 2007 International Press

Adv. Theor. Math. Phys. 11 (2007) 91-146

\title{
Fermion systems in discrete
}

space-time - outer symmetries

and spontaneous

symmetry breaking

\author{
Felix Finster
}

NWF I-Mathematik, Universität Regensburg, 93040 Regensburg, Germany, Felix.Finster@mathematik.uni-regensburg.de

\begin{abstract}
A systematic procedure is developed for constructing fermion systems in discrete space-time which have a given outer symmetry. The construction is illustrated by simple examples. For the symmetric group, we derive constraints for the number of particles. In the physically interesting case of many particles and even more space-time points, this result shows that the permutation symmetry of discrete space-time is always spontaneously broken by the fermionic projector.
\end{abstract}

Contents

1. Discrete fermion systems with outer symmetry 2

2. Reduction of the proper free gauge group 3

e-print archive: http://lanl.arXiv.org/abs/hep-th/0601039 
3. A decomposition of $\mathrm{U}(\sigma) \quad 16$

4. Simple systems and simple subsystems 26

5. A representation of a group extension of $\mathcal{N} \quad 33$

6. The pinned symmetry group 35

7. Building up general systems: a constructive procedure 37

8. Examples: abelian outer symmetries and lattices 38

9. Spontaneous breaking of the permutation symmetry 43

References $\quad 55$

\section{Discrete fermion systems with outer symmetry}

We briefly recall the mathematical setting of the fermionic projector in discrete space-time as introduced in [1] (see also [2] or [3]). Let $H$ be a finite-dimensional complex vector space endowed with a non-degenerate symmetric sesquilinear form $\langle. \mid$.$\rangle . We call (H,\langle. \mid\rangle$.$) an indefinite inner product$ space. To every element $x$ of a finite set $M=\{1, \ldots, m\}$, we associate a projector $E_{x}$. We assume that these projectors are orthogonal and complete,

$$
E_{x} E_{y}=\delta_{x y} E_{x}, \quad \sum_{x \in M} E_{x}=\mathbb{1}
$$

and that the images of the $E_{x}$ are non-degenerate subspaces of $H$. We denote the signature of the subspace $E_{x}(H) \subset H$ by $\left(p_{x}, q_{x}\right)$ and refer to it as the spin dimension at $x$. We call the structure $\left(H,\langle. \mid\rangle,.\left(E_{x}\right)_{x \in M}\right)$ discrete space-time. $M$ are the discrete space-time points and $E_{x}$ the spacetime projectors. The fermionic projector $P$ is defined as a projector on a subspace of $H$ which is negative definite and of dimension $f$. The vectors in the image of $P$ have the interpretation as the quantum states of the particles of the system and $f$ is the number of particles. In what follows, we refer to $\left(H,\langle. \mid\rangle,.\left(E_{x}\right)_{x \in M}, P\right)$ as a fermion system in discrete space-time or, for brevity, a discrete fermion system.

We point out that in $[1,2]$ we assumed furthermore that the spin dimension is equal to $(n, n)$ at every space-time point. Here we consider a more general spin dimension $\left(p_{x}, q_{x}\right)$ for two reasons. First, a constant spin dimension $(n, n)$ would not be a major simplification for what follows. Second, 
even if we started with constant spin dimension $(n, n)$, the corresponding simple systems (see Section 4) will, in general, have a spin dimension which varies in space-time, and therefore it is more elegant to begin right away with a non-constant spin dimension $\left(p_{x}, q_{x}\right)$.

In this paper, we consider discrete fermion systems which have a spacetime symmetry, as described by the next definition. We denote the symmetric group of $M$ (= the group of all permutations of $M)$ by $\mathcal{S}_{m}$.

Definition 1.1. A subgroup $\mathcal{O}$ of the symmetric group $\mathcal{S}_{m}$ is called outer symmetry group of the discrete fermion system if for every $\sigma \in \mathcal{O}$ there is a unitary transformation $U$ such that

$$
U P U^{-1}=P \quad \text { and } \quad U E_{x} U^{-1}=E_{\sigma(x)} \forall x \in M .
$$

Our aim is to characterize the discrete fermion systems for a given outer symmetry group $\mathcal{O}$.

\section{Reduction of the proper free gauge group}

The transformation $U$ in Definition 1.1 is determined only up to transformations which leave both the fermionic projector and the space-time projectors invariant, i.e.,

$$
U P U^{-1}=P \quad \text { and } \quad U E_{x} U^{-1}=E_{x} \quad \forall x \in M .
$$

In simple terms, our aim is to "fix" such transformations, thereby making the transformation $U$ in (1.2) unique. This is desirable because the resulting mapping $\sigma \mapsto U(\sigma)$ would be a representation of the outer symmetry group on $H$, making it possible to apply the representation theory for finite groups. Before entering the problem of fixing the transformations (2.1), we need to study these transformations in detail.

As in [2], we introduce the gauge group $\mathcal{G}$ as the group of all unitary transformations $U$ which leave discrete space-time invariant, i.e.,

$$
U E_{x} U^{-1}=E_{x} \quad \forall x \in M .
$$

A transformation of the fermionic projector

$$
P \longrightarrow U P U^{-1} \text { with } U \in \mathcal{G}
$$

is called a gauge transformation. Clearly, the transformations (2.1) are gauge transformations and they form the following subgroup of $\mathcal{G}$. 
Definition 2.1. We define the free gauge group $\mathcal{F}$ by

$$
\mathcal{F}=\left\{U \in \mathcal{G} \text { with } U P U^{-1}=P\right\} .
$$

The free gauge group describes symmetries of the fermionic projector which do not involve a transformation of the space-time points and which are therefore sometimes referred to as inner symmetries. Unfortunately, representations of the free gauge group are, in general, not completely reducible, as the following example shows.

Example 2.2. Consider the case $m=2$, spin dimension $(1,1)$ and $f=1$. As in [2], we represent the scalar product $\langle$.$| . \rangle$ with a signature matrix $S$. More specifically,

$$
\langle u \mid v\rangle=(u \mid S v) \quad \forall u, v \in H,
$$

where (.|.) denotes the canonical scalar product on $\mathbb{C}^{4}$ and $S=S^{\dagger}, S^{2}=\mathbb{1}$. By choosing a suitable basis, we can arrange that

$$
S=\left(\begin{array}{cccc}
0 & 1 & 0 & 0 \\
1 & 0 & 0 & 0 \\
0 & 0 & 1 & 0 \\
0 & 0 & 0 & -1
\end{array}\right), \quad E_{1}=\left(\begin{array}{cc}
\mathbb{1} & 0 \\
0 & 0
\end{array}\right), \quad E_{2}=\left(\begin{array}{ll}
0 & 0 \\
0 & \mathbb{1}
\end{array}\right)
$$

where for $E_{1 / 2}$ we used a block matrix notation (thus every matrix entry stands for a $2 \times 2$ matrix). We represent the fermionic projector in braket notation as

$$
P=-|u\rangle\langle u| \text { with }\langle u \mid u\rangle=-1 .
$$

We choose $u=(1,0,0,1)$. The free gauge group consists of all gauge transformations $U$ which change $u$ at most by a phase. A short calculation yields that such $U$ are precisely of the form

$$
U=\mathrm{e}^{\mathrm{i} \alpha}\left(\begin{array}{cccc}
1 & \mathrm{i} \gamma & 0 & 0 \\
0 & 1 & 0 & 0 \\
0 & 0 & \mathrm{e}^{\mathrm{i} \beta} & 0 \\
0 & 0 & 0 & 1
\end{array}\right) \quad \text { with } \alpha, \beta, \gamma \in \mathbb{R}
$$

Hence $\mathcal{F}$ is group isomorphic to $S^{1} \times S^{1} \times \mathbb{R}$ (where $\mathbb{R}$ denotes the additive group $(\mathbb{R},+))$. The subspace spanned by the vector $(1,0,0,0)$ is invariant, but it has no invariant complement (this is indeed quite similar to the standard example of the triangular matrices as mentioned, for example, in [6, Section 2.2]). Hence the group representation (2.3) is not completely reducible. 
Our method for avoiding this problem is to take the quotient by the subgroup of the free gauge group which leaves every vector of $P(H)$ invariant.

Definition 2.3. The trivial gauge group $\mathcal{F}_{0}$ is defined by

$$
\mathcal{F}_{0}=\{U \in \mathcal{G} \text { with } U P=P\} .
$$

Taking the adjoint of the relation $U P=P$, we find that $P=P U^{-1}$ and thus $U P U^{-1}=U P=P$, showing that $\mathcal{F}_{0}$ is really a subgroup of $\mathcal{F}$. Furthermore, for every $g \in \mathcal{F}$,

$$
g \mathcal{F}_{0} g^{-1} P=g \mathcal{F}_{0} g^{-1} P^{2}=g\left(\mathcal{F}_{0} P\right) g^{-1} P=g P g^{-1} P=P,
$$

proving that $g \mathcal{F}_{0} g^{-1} \subset \mathcal{F}_{0}$. Hence $\mathcal{F}_{0}$ is a normal subgroup and we can form the quotient group.

Definition 2.4. The proper free gauge group $\hat{\mathcal{F}}$ is defined by

$$
\hat{\mathcal{F}}=\mathcal{F} / \mathcal{F}_{0} .
$$

In order to make $\hat{\mathcal{F}}$ a metric space, we introduce the distance function

$$
d(\hat{g}, \hat{h})=\inf _{g, h \in \mathcal{F}}\|g-h\|_{H},
$$

where $g$ and $h$ run over all representatives of $\hat{g}, \hat{h} \in \hat{\mathcal{F}}$ and $\|\cdot\|_{H}$ is the sup-norm corresponding to a given norm on $H$. We remark that the topology generated by this metric coincides with the quotient topology.

Example 2.5. In the setting of Example 2.2, $\mathcal{F}_{0}$ consists of all unitary transformations $U$ of the form (2.3) with $\alpha=0$. Hence the equivalence class $\hat{U}$ corresponding to a unitary transformation of the form (2.3) is the set

$$
\hat{U}=\left\{\mathrm{e}^{\mathrm{i} \alpha}\left(\begin{array}{cccc}
1 & \mathrm{i} \gamma & 0 & 0 \\
0 & 1 & 0 & 0 \\
0 & 0 & \mathrm{e}^{\mathrm{i} \beta} & 0 \\
0 & 0 & 0 & 1
\end{array}\right) \text { with } \beta, \gamma \in \mathbb{R}\right\}
$$

These equivalence classes are described completely by the parameter $\alpha$ and thus $\hat{\mathcal{F}}$ is group isomorphic to $U(1)$. Moreover, it is easy to verify that the topology induced by the norm (2.4) coincides with the standard topology of $U(1)$. Hence we can identify $\hat{\mathcal{F}}$ with the compact Lie group $U(1)$. This group can be obtained even without forming equivalence classes simply by restricting $U$ to the image of $P$, because

$$
U_{\mid P(H)}=\mathrm{e}^{\mathrm{i} \alpha} \mathbb{1}_{P(H)} .
$$


The last example illustrates and motivates the following general constructions. It will be crucial that $I:=P(H)$ is a definite subspace of $H$. Thus the inner product $\langle. \mid$.$\rangle makes I$ a Hilbert space. We denote the corresponding norm by

$$
\|u\|_{I}:=\sqrt{-\langle u \mid u\rangle}
$$

Furthermore, we denote the unitary endomorphisms of $I$ by $U(I)$. Choosing an orthonormal basis of $I$, one sees that $U(I)$ can be identified with the compact Lie group $U(f)$. The condition $P=U P U^{-1}$ in Definition 2.1 means that every $U \in \mathcal{F}$ maps $I$ to itself, and thus the restriction to $I$ gives a mapping

$$
\varphi: \mathcal{F} \longrightarrow \mathrm{U}(I): U \longmapsto U_{\mid I}
$$

Since every $U_{0} \in \mathcal{F}_{0}$ is trivial on $I$, the mapping $\varphi$ is well defined on the equivalence classes $\mathcal{F} / \mathcal{F}_{0}$. Furthermore, $\varphi\left(U^{\prime}\right)=\varphi(U)$ if and only if $U^{\prime} U^{-1}$ $\in \mathcal{F}_{0}$. Thus $\varphi$ gives rise to the injection

$$
\varphi: \hat{\mathcal{F}} \hookrightarrow U(I) .
$$

Since every free gauge transformation $U \in \mathcal{F}$ maps the subspaces $E_{x}(H)$ into themselves, the corresponding $\varphi(U) \in U(I)$ is locally unitary in the following sense.

Definition 2.6. A linear map $U \in \mathrm{U}(I)$ is called locally unitary if for all $u, v \in I$ and all $x \in M$ the following conditions are satisfied:

(i) $E_{x} v=0 \Longleftrightarrow E_{x} U v=0$.

(ii) $\left\langle E_{x} U u \mid U v\right\rangle=\left\langle E_{x} u \mid v\right\rangle$.

The group of all locally unitary transformations is denoted by $U_{\text {loc }}(I)$.

Lemma 2.7. The group $U_{\mathrm{loc}}(I)$ is a compact Lie subgroup of $U(I)$.

Proof. Let $\mathcal{A}$ be the set of all symmetric operators $A$ on $I$ which satisfy for all $u, v \in I$ and $x \in M$ the conditions

$$
E_{x} v=0 \Longleftrightarrow E_{x} A v=0 \text { and }\left\langle E_{x} A u \mid v\right\rangle=\left\langle E_{x} u \mid A v\right\rangle .
$$

Obviously, $\mathcal{A}$ is a linear subspace of $\operatorname{End}(I)$ (where $\operatorname{End}(I)$ denotes the linear mappings of $I$ to itself). Furthermore, the above conditions are compatible with the Lie bracket $\{A, B\}=\mathrm{i}[A, B]$, and thus $\mathcal{A}$ is a Lie algebra. The exponential map $A \mapsto \exp (\mathrm{i} A)$ maps $\mathcal{A}$ into $U_{\text {loc }}(I)$. In a neighborhood 
of $\mathbb{1} \in U(I)$, we can define the logarithm by the power series

$$
\log (V)=\log (\mathbb{1}-(\mathbb{1}-V))=-\sum_{n=1}^{\infty} \frac{(\mathbb{1}-V)^{n}}{n},
$$

showing that the exponential map is locally invertible near $0 \in \mathcal{A}$. Hence the exponential map gives a chart near $\mathbb{1} \in U_{\text {loc }}(I)$. Using the group structure, we can "translate" this chart to the neighborhood of any $\hat{V} \in U_{\text {loc }}(I)$ to get a smooth atlas. We conclude that $U_{\text {loc }}(I)$ is a Lie subgroup of $U(I)$. Finally, the conditions (i) and (ii) in Definition 2.6 are preserved if one takes limits, proving that $U_{\text {loc }}(I)$ is closed in $U(I)$ and thus compact.

The construction of the next lemma allows us to extend every locally unitary map to a free gauge transformation on $H$.

Lemma 2.8. (Extension lemma) There is a constant $C>0$ (depending only on $I$ and the norm $\left.\|\cdot\|_{H}\right)$ such that for every locally unitary $U \in U(I)$ there is a $V \in \mathcal{F}$ with $\varphi(V)=U$ and

$$
\|\mathbb{1}-V\|_{H} \leq C\|\mathbb{1}-U\|_{I} .
$$

This $V$ can be chosen to depend smoothly on $U$, giving rise to a smooth injection

$$
\lambda: U_{\text {loc }}(I) \hookrightarrow \mathcal{F} \subset \operatorname{End}(H),
$$

which is a group homomorphism.

Proof. The first step is to "localize" $U$ at a given $x \in M$ to obtain an operator

$$
U_{x}: E_{x}(I) \longrightarrow E_{x}(I) .
$$

Introducing the abbreviations $I_{x}:=E_{x}(I)$ and $H_{x}:=E_{x}(H)$, we choose an injection $\iota_{x}: I_{x} \hookrightarrow I$ such that

$$
E_{x} \iota_{x}=\mathbb{1}_{I_{x}} .
$$

We define $U_{x}$ by

$$
U_{x}=E_{x} U \iota_{x}: I_{x} \longrightarrow I_{x} .
$$

Let us verify that this definition is independent of the choice of $\iota_{x}$. For two different injections $\iota_{x}$ and $\iota_{x}^{\prime}$, we know from (2.8) that for all $u_{x} \in I_{x}$,

$$
E_{x}\left(\iota_{x}-\iota_{x}^{\prime}\right) u_{x}=0 .
$$

Using that $U$ is locally unitary, we conclude from condition (i) in Definition 2.6 that

$$
0=E_{x} U\left(\iota_{x}-\iota_{x}^{\prime}\right) u_{x}=\left(U_{x}-U_{x}^{\prime}\right) u_{x} .
$$


Let us collect some properties of $U_{x}$. First of all, choosing for a given $u \in I$ the injection $\iota_{x}^{\prime}$ such that $\iota_{x}^{\prime} E_{x} u=u$, the above independence of $U_{x}$ of the choice of the injection implies that for all $u \in I$,

$$
E_{x} U u=E_{x} U \iota_{x}^{\prime} E_{x} u=U_{x} E_{x} u,
$$

and thus for all $u_{x} \in I_{x}$,

$$
E_{x} U \iota_{x} u_{x}=U_{x} u_{x}
$$

As a consequence,

$$
\left(U^{-1}\right)_{x} U_{x} u_{x} \stackrel{(2.10)}{=}\left(U^{-1}\right)_{x} E_{x} U \iota_{x} u_{x} \stackrel{(2.9)}{=} E_{x} U^{-1} U \iota_{x} u_{x}=u_{x}
$$

In a more compact notation,

$$
\left(U_{x}\right)^{-1}=\left(U^{-1}\right)_{x}
$$

and thus it is unambiguous to simply write $U_{x}^{-1}$. By restriction, we can also consider the norm $\|\cdot\|_{H}$ on the subspace $H_{x}$. Since every unitary map in the Hilbert space $I$ has norm 1, we can estimate the corresponding norm of $U_{x}$ by

$$
\left\|U_{x}\right\|_{H} \leq\left\|E_{x}\right\|\|U\|_{I}\left\|\iota_{x}\right\|=\left\|E_{x}\right\|\left\|\iota_{x}\right\| .
$$

Note that the resulting upper bound is independent of $U_{x}$. Applying the same argument to $U_{x}^{-1}$, we conclude that there is a constant $c$ independent of $U_{x}$ such that

$$
\left\|U_{x}\right\|_{H}+\left\|U_{x}^{-1}\right\|_{H} \leq c .
$$

Furthermore, we have

$$
\begin{aligned}
\left\|\mathbb{1}-U_{x}\right\| & =\left\|E_{x}(\mathbb{1}-U) \iota_{x}\right\| \leq c\|\mathbb{1}-U\|, \\
\left\|\mathbb{1}-U_{x}^{-1}\right\| & \leq\left\|U_{x}^{-1}\right\|\left\|U_{x}-\mathbb{1}\right\| \leq c^{2}\|\mathbb{1}-U\| .
\end{aligned}
$$

Finally, $U_{x}$ is isometric on $I_{x}$. Namely, using the properties of the spacetime projectors together with condition (ii) in Definition 2.6, we obtain that 
for all $u_{x}, v_{x} \in I_{x}$,

$$
\left\langle U_{x} u_{x} \mid U_{x} v_{x}\right\rangle=\left\langle E_{x} U \iota_{x} u_{x} \mid U \iota_{x} v_{x}\right\rangle=\left\langle E_{x} \iota_{x} u_{x} \mid \iota_{x} v_{x}\right\rangle=\left\langle u_{x} \mid v_{x}\right\rangle
$$

Our goal is to construct a unitary operator $V_{x}: H_{x} \rightarrow H_{x}$ which coincides on $I_{x}$ with $U_{x}$ and satisfies the inequality

$$
\left\|\mathbb{1}-V_{x}\right\|_{H} \leq C\|\mathbb{1}-U\|_{I}
$$

Namely, provided that the operator $V_{x}$ can be constructed for every $x \in M$, we can construct $V$ by taking

$$
V=\sum_{x \in M} V_{x} E_{x}: H \longrightarrow H
$$

This operator is obviously unitary and invariant on the subspaces $H_{x}$, and thus $V \in \mathcal{F}$. Furthermore, for all $x \in M$ and $u \in I$,

$$
E_{x} \varphi(V) u=E_{x} V u=E_{x} V_{x} E_{x} u=E_{x} U_{x} E_{x} u \stackrel{(2.9)}{=} E_{x} U u
$$

proving that $\varphi(V)=U$. Hence $V$ really has all the required properties.

In order to construct $V_{x}$, we choose in $I_{x}$ a non-degenerate subspace of maximum dimension and in this subspace a pseudo-orthonormal basis $e_{i}$. We extend this basis by vectors $f_{j}$ to a basis of $I_{x}$ (thus the vectors $f_{j}$ are all null and orthogonal to $I_{x}$ ). Next we choose vectors $h_{j} \in H_{x}$ which are orthogonal to the $e_{i}$ and conjugate to the $f_{j}$ in the sense that $\left\langle f_{i} \mid h_{j}\right\rangle=\delta_{i j}$. Then the span of the vectors $e_{i}, f_{j}$ and $h_{j}$ is non-degenerate, and we can choose on its orthogonal complement a pseudo-orthonormal basis $g_{k}$. We thus obtain a basis $\left(e_{i}, f_{j}, g_{k}, h_{j}\right)$ of $H_{x}$. Using a block matrix notation in this basis, the signature matrix takes the form

$$
S=\left(\begin{array}{cccc}
S_{1} & 0 & 0 & 0 \\
0 & 0 & 0 & \mathbb{1} \\
0 & 0 & S_{2} & 0 \\
0 & \mathbb{1} & 0 & 0
\end{array}\right)
$$

where $S_{1}$ and $S_{2}$ are diagonal matrices with entries equal to \pm 1 . Without loss of generality, we choose the norm on $H_{x}$ such that it coincides, in this basis, with the standard Euclidean norm on $\mathbb{C}^{p_{x}+q_{x}}$. 
We represent operators on $I_{x}$ as $2 \times 2$ block matrices in the basis $\left(e_{i}, f_{j}\right)$, e.g.,

$$
U_{x}=\left(\begin{array}{cc}
W & X \\
C & A
\end{array}\right) .
$$

Since $U_{x}$ is isometric on $I_{x}$, we find

$$
\left\langle e_{i} \mid e_{j}\right\rangle=\left\langle U_{x} e_{i} \mid U_{x} e_{j}\right\rangle=\left\langle W e_{i} \mid W e_{j}\right\rangle,
$$

showing that $W$ is unitary in the sense that $W^{-1}=S_{1} W^{\dagger} S_{1}$. Furthermore,

$$
0=\left\langle e_{i} \mid f_{j}\right\rangle=\left\langle U_{x} e_{i} \mid U_{x} f_{j}\right\rangle=\left\langle W e_{i} \mid X f_{j}\right\rangle,
$$

and since $W$ is unitary, we conclude that $X$ must vanish identically. Arguing similarly for $U_{x}^{-1}$ and using that $U_{x}$ and $U_{x}^{-1}$ are inverse to each other, one easily verifies that they must be of the form

$$
U_{x}=\left(\begin{array}{cc}
W & 0 \\
C & A
\end{array}\right), \quad U_{x}^{-1}=\left(\begin{array}{cc}
W^{-1} & 0 \\
D & A^{-1}
\end{array}\right),
$$

where $D=-A^{-1} C W^{-1}$. We choose $V_{x}$ as

$$
V_{x}=\left(\begin{array}{cccc}
W & 0 & 0 & S_{1} D^{\dagger} \\
C & A & 0 & B \\
0 & 0 & 1 & 0 \\
0 & 0 & 0 & \left(A^{-1}\right)^{\dagger}
\end{array}\right) \quad \text { with } B=-\frac{1}{2} A D S_{1} D^{\dagger} .
$$

Obviously, $V_{x}$ coincides on $I_{x}$ with $U_{x}$ and a direct calculation shows that $V_{x}$ is unitary on $H_{x}$, i.e.,

$$
V_{x} S V_{x}^{\dagger} S=\mathbb{1} .
$$

Using that, according to (2.11), the norms of all the matrix entries appearing in $(2.15)$ can be estimated in terms of $c$, we find that

$$
\begin{aligned}
\left\|\mathbb{1}-V_{x}\right\| & \leq\left(1+c^{2}\right)\left(\|\mathbb{1}-W\|+\|\mathbb{1}-A\|+\left\|\mathbb{1}-A^{-1}\right\|+\|C\|+\|D\|\right) \\
& \leq\left(1+c^{2}\right)\left(\left\|\mathbb{1}-U_{x}\right\|+\left\|\mathbb{1}-U_{x}^{-1}\right\|\right) .
\end{aligned}
$$

Applying (2.12) and (2.13) give the desired inequality (2.14).

Finally, it is obvious from the explicit formulas (2.15) and (2.16) that our choice of $V$ depends smoothly on $U$ and that the mapping $\lambda$ is a group homomorphism.

The last lemma shows, in particular, that (2.5) gives a one-to-one correspondence between proper free gauge transformations and locally unitary transformations. Since $U_{\text {loc }}(I)$ is a compact Lie group, one might expect that $\hat{\mathcal{F}}$ is itself compact. This is really the case, as we now prove. 
Lemma 2.9. The proper free gauge group $\hat{\mathcal{F}}$ is a compact Lie group. The mapping

$$
\varphi: \hat{\mathcal{F}} \longrightarrow U_{\text {loc }}(I)
$$

is a Lie group homomorphism.

Proof. We first consider the infinitesimal generators of the groups. We thus introduce the following families of linear operators on $H$,

$$
\begin{aligned}
& \mathcal{A}=\left\{A \text { with } A^{*}=A,\left[A, E_{x}\right]=0 \forall x \in M \text { and }[A, P]=0\right\}, \\
& \mathcal{A}_{0}=\left\{A \text { with } A^{*}=A,\left[A, E_{x}\right]=0 \forall x \in M \text { and } A P=0\right\} .
\end{aligned}
$$

Obviously, these families are linear subspaces of $\operatorname{End}(H)$ which, together with the Lie bracket $\{A, B\}=\mathrm{i}[A, B]$, form real Lie algebras. Furthermore, $\mathcal{A}_{0}$ is a subalgebra of $\mathcal{A}$, and the calculation

$$
\left[A_{0}, A\right] P=A_{0} A P-A A_{0} P=\left(A_{0} P\right) A-A\left(A_{0} P\right)=0
$$

shows that $\mathcal{A}_{0}$ is an ideal of $\mathcal{A}$. Hence $\hat{\mathcal{A}}:=\mathcal{A} / \mathcal{A}_{0}$ is again a Lie algebra. (Since $\hat{\mathcal{A}}$ is a finite-dimensional vector space, we need not worry about introducing a norm or topology on it.)

The exponential map $a \mapsto \exp (\mathrm{i} a)$ gives a mapping from $\hat{\mathcal{A}}$ to $\hat{\mathcal{F}}$ which is obviously continuous. Assume conversely that $\hat{V} \in B_{\varepsilon}(\mathbb{1}) \subset \hat{\mathcal{F}}$ (corresponding to the distance function (2.4)). Since restricting an operator on $H$ to the subspace $I$ decreases its norm, we know that for any representative $V \in \mathcal{F}$ of $\hat{V}$,

$$
\|\mathbb{1}-\varphi(\hat{V})\|_{I} \leq c\|\mathbb{1}-V\|_{H}
$$

(with $c$ independent of $\hat{V}$ and $V$ ), and taking the infimum over all representatives, we find that

$$
\|\mathbb{1}-\varphi(\hat{V})\|_{I} \leq c \varepsilon
$$

Since the map $\varphi(\hat{V})$ is locally unitary, Lemma 2.8 allows us to choose a representative $V$ of $\hat{V}$ satisfying the inequality

$$
\|\mathbb{1}-V\|_{H} \leq C c \varepsilon .
$$

Hence, after choosing $\varepsilon$ sufficiently small, the logarithm of $V$ may again be defined by the power series (2.6). We conclude that the exponential map is invertible locally near $0 \in \hat{\mathcal{A}}$ and that its inverse is continuous. Hence the exponential map gives a chart near $\mathbb{1} \in \hat{\mathcal{F}}$. Using the group structure, we get a smooth atlas. We conclude that $\hat{\mathcal{F}}$ is a Lie group. 
According to Lemma 2.8, the image of $\varphi$ consists precisely of all locally unitary maps, which by Lemma 2.7 form a closed subset of $U(I)$. Furthermore, restricting the above exponential map to $I$,

$$
\varphi \exp (\mathrm{i} a)=\exp \left(\mathrm{i} a_{\mid I}\right),
$$

we obtain precisely the chart near $\mathbb{1} \in U_{\text {loc }}(I)$ constructed in Lemma 2.7. Hence $\varphi$ is a smooth map from $\hat{\mathcal{F}}$ to $U_{\text {loc }}(I)$. Its inverse can be written as $\varphi^{-1}=\pi \lambda$ with $\lambda$ as given by $(2.7)$ and $\pi: \mathcal{F} \rightarrow \hat{\mathcal{F}}$ the natural projection. Hence the smoothness of $\varphi^{-1}$ follows from the smoothness of $\lambda$.

The previous lemma allows us to identify $\hat{\mathcal{F}}$ with the compact subgroup $U_{\text {loc }}(I)$ of $U(I)$. As the next lemma shows, compactness implies complete reducibility into definite subspaces.

Lemma 2.10. Let $\mathcal{E}$ be a finite group or a compact Lie group, and $U$ a unitary representation of $\mathcal{E}$ on an indefinite inner product space $H$ of signature $(p, q)$. Then $H$ can be decomposed into a direct sum of irreducible subspaces, which are all definite and mutually orthogonal.

Proof. We introduce on $(H,\langle. \mid\rangle$.$) , in addition, a positive-definite scalar prod-$ uct (.|.). By averaging over the group,

$$
(u \mid v)_{\mathcal{E}}:= \begin{cases}\frac{1}{\# \mathcal{E}} \sum_{g \in \mathcal{E}}(U(g) u \mid U(g) v) & \text { if } \mathcal{E} \text { is a finite group } \\ \frac{1}{|\mathcal{E}|} \int_{\mathcal{E}}(U(g) u \mid U(g) v) d g & \text { if } \mathcal{E} \text { is a compact Lie group }\end{cases}
$$

we obtain an invariant scalar product $(. \mid \cdot)_{\mathcal{E}}$. Hence the representation $U$ is unitary with respect to both $\langle. \mid$.$\rangle and (. \mid .)_{\mathcal{E}}$.

In a suitable basis, $(. \mid \cdot)_{\mathcal{E}}$ coincides with the Euclidean scalar product on $\mathbb{C}^{p+q}$, whereas $\langle. \mid$.$\rangle takes the form$

$$
\langle u \mid v\rangle=(u \mid S v)_{\mathcal{E}} \quad \text { with } S=\operatorname{diag}(\underbrace{1, \ldots, 1}_{p \text { times }}, \underbrace{-1, \ldots,-1}_{q \text { times }}) .
$$

Let $H^{+} \subset H$ be the positive-definite subspace of all vectors whose last $q$ components vanish. Then for every $v \in H^{+}$and every representation matrix $U=U(g)$,

$$
\begin{aligned}
& \sum_{i=1}^{p}\left|v^{i}\right|^{2}=(v \mid v)_{\mathcal{E}}=(U v \mid U v)_{\mathcal{E}}=\sum_{i=1}^{p+q}\left|(U v)^{i}\right|^{2} \\
& \sum_{i=1}^{p}\left|v^{i}\right|^{2}=\langle v \mid v\rangle=\langle U v \mid U v\rangle=\sum_{i=1}^{p}\left|(U v)^{i}\right|^{2}-\sum_{i=p+1}^{p+q}\left|(U v)^{i}\right|^{2} .
\end{aligned}
$$


Subtracting the two lines, we find that

$$
2 \sum_{i=p+1}^{p+q}\left|(U v)^{i}\right|^{2}=0,
$$

and thus $U v \in H^{+}$. We conclude that $H^{+}$is an invariant subspace.

Similarly, the subspace $H^{-}$of all vectors, whose first $p$ components vanish, is also invariant. In this way, we have decomposed $H$ into an orthogonal direct sum of two invariant definite subspaces. We finally decompose these invariant definite subspaces in the standard way into mutually orthogonal, irreducible subspaces.

We are now ready to prove the main result of this section. We always endow the tensor product $\mathbb{C}^{l} \otimes H$ (where $H$ is an inner product space) with the natural inner product

$$
\left\langle\left(u_{i}\right) \mid\left(v_{j}\right)\right\rangle=\sum_{i=1}^{l}\left\langle u_{i} \mid v_{i}\right\rangle_{H} .
$$

Theorem 2.11. There are integers $\left(l_{r}\right)_{r=1, \ldots, R}$,

$$
1 \leq l_{1} \leq \cdots \leq l_{R}
$$

such that $\hat{\mathcal{F}}$ is Lie group isomorphic to the product of the corresponding unitary groups,

$$
\hat{\mathcal{F}} \simeq U\left(l_{1}\right) \times \cdots \times U\left(l_{R}\right) .
$$

The inner product space $(H,\langle. \mid\rangle$.$) is isomorphic to the orthogonal direct sum$

$$
H \simeq H^{(0)} \oplus\left(\bigoplus_{r=1}^{R} \mathbb{C}^{l_{r}} \otimes H^{(r)}\right)
$$

where $H^{(r)}$ are inner product spaces of signature $\left(p^{(r)}, q^{(r)}\right)$. Under the isomorphism (2.21), the projectors $P$ and $\left(E_{x}\right)_{x \in M}$ take the form

$$
\begin{gathered}
P \simeq 0 \oplus\left(\bigoplus_{r=1}^{R} \mathbb{1}_{\mathbb{C}^{l_{r}}} \otimes P^{(r)}\right), \\
E_{x} \simeq E_{x}^{(0)} \oplus\left(\bigoplus_{r=1}^{R} \mathbb{1}_{\mathbb{C}^{l_{r}}} \otimes E_{x}^{(r)}\right),
\end{gathered}
$$

where $P^{(r)}$ and $E_{x}^{(r)}$ are projectors on $H^{(r)}$. None of the operators $P^{(r)}$ vanishes. Furthermore, $\hat{\mathcal{F}}$ acts only on the factors $\mathbb{C}^{l_{r}}$ in the sense that for 
every representative $V \in \mathcal{F}$ of a $\hat{V}=\left(V_{1}, \ldots, V_{R}\right) \in \hat{\mathcal{F}}$,

$$
V_{\mid I}=\bigoplus_{r=1}^{R} V_{r} \otimes \mathbb{1}_{I^{(r)}},
$$

where we set $I=P(H)$ and $I^{(r)}=P^{(r)}\left(H^{(r)}\right)$.

Choosing $H^{(0)}$ maximal in the sense that every subspace $J \subset H$ satisfies the condition

$$
J \text { definite, } P(J)=0 \text { and } \quad E_{x}(J) \subset J \forall x \in M \Longrightarrow J \subset H^{(0)},
$$

the above representation is unique.

Note that we do not exclude the case $p^{(0)}=0=q^{(0)}$ and thus $H^{(0)}$ might be zero-dimensional. The situation is different if $r \geq 1$, because in this case we know that $P^{(r)}$ does not vanish, and therefore the dimension of $H^{(r)}$ must be at least one, $p^{(r)}+q^{(r)} \geq 1$. We also point out that $P$ vanishes on $H^{(0)}$ and thus $I^{(0)}=\{0\}$; this is why in (2.24) we could leave out the direct summand corresponding to $H^{(0)}$.

Proof of Theorem 2.11. The mapping $\lambda \circ \varphi$ (with $\varphi$ and $\lambda$ according to (2.17) and (2.7), respectively) is a unitary representation of $\hat{\mathcal{F}}$ on $H$. According to Lemma 2.10, this representation splits into irreducible representations on definite, mutually orthogonal subspaces. We denote the appearing nontrivial, non-equivalent irreducible representations by $V_{1}, \ldots$ $V_{R}$ and let $V_{0}$ be the trivial representation on $\mathbb{C}$. We let these irreducible representations act unitarily on the respective vector spaces $\mathbb{C}^{l_{r}}, l_{r} \geq 1$, endowed with the standard Euclidean scalar product. Collecting the direct summands of $H$ corresponding to equivalent irreducible representations, we obtain an orthogonal decomposition of the form

$$
H \simeq \bigoplus_{r=0}^{R} \mathbb{C}^{l_{r}} \otimes H^{(r)}
$$

with inner product spaces $H^{(r)}$ of signature $\left(p^{(r)}, q^{(r)}\right)$ together with the representation

$$
(\lambda \circ \varphi)(g)=\bigoplus_{r=0}^{R} V_{r}(g) \otimes \mathbb{1}_{H^{(r)}} \quad \forall g \in \hat{\mathcal{F}} .
$$

Schur's lemma yields that the operators $P$ and $E_{x}$ take the form

$$
P \simeq \bigoplus_{r=0}^{R} \mathbb{1}_{\mathbb{C}^{r}} \otimes P^{(r)}, \quad E_{x} \simeq \bigoplus_{r=0}^{R} \mathbb{1}_{\mathbb{C}^{r}} \otimes E_{x}^{(r)} .
$$


By restricting to $I,(2.27)$ gives

$$
\varphi(g) \simeq \bigoplus_{r=0}^{R} V_{r}(g) \otimes \mathbb{1}_{I^{(r)}},
$$

and according to Lemma 2.9 this is simply the fundamental representation of $U_{\text {loc }}(I)$.

Suppose that $P^{(r)}=0$. Then replacing $V_{r}$ by the trivial representation, we get a new group homomorphism $\tilde{\lambda}: \hat{\mathcal{F}} \hookrightarrow \mathcal{F}$ with $\varphi \circ \tilde{\lambda}=\tilde{\lambda}_{\mid I}=\lambda_{\mid I}=\mathbb{1}$, for which the above construction applies just as well. Then $H^{(r)}$ will be combined with $H^{(0)}$. In this way, we can arrange that $P^{(r)} \neq 0$ unless $r=0$.

Using the representations (2.26) and (2.28), it is obvious that every transformation of the form

$$
\bigoplus_{r=0}^{R} U_{r} \otimes \mathbb{1}_{I^{(r)}} \quad \text { with } U_{r} \in U\left(l_{r}\right)
$$

is locally unitary. Comparing with (2.24), one sees that the $V_{r}(g)$ can be chosen independently and arbitrarily in $U\left(l_{r}\right)$. However, one must keep in mind that if $I^{(r)}=\{0\}$, the corresponding summand drops out of both (2.24) and (2.29). We conclude that $U_{\text {loc }}$ coincides with the product of all those groups $U\left(l_{r}\right)$ for which $I^{(r)} \neq\{0\}$. This implies that $P^{(0)}$ must vanish, because otherwise $V_{0}=U\left(l_{0}\right)$ would be a non-trivial representation. After reordering the $l_{r}$, we obtain $(2.20)$ as well as the desired representations $(2.21)-(2.24)$.

It is obvious that every subspace $J$ which satisfies the conditions on the left of $(2.25)$ can be combined with $H^{(0)}$. The only arbitrariness in the construction is the choice of the embedding $\lambda$. Choosing $H^{(0)}$ maximal corresponds to choosing $\lambda$ equal to the identity on a non-degenerate subspace of maximal dimension. Then the signature of each subspace $E_{x} \lambda(\hat{\mathcal{F}})$ coincides with the signature of the smallest non-degenerate subspace containing $I_{x}$ and is therefore fixed. As a consequence, two different choices of $\lambda$ can be related to each other by a free gauge transformation. This proves uniqueness of our representation.

We denote the signature of $E_{x}^{(r)}\left(P^{(r)}\right)$ by $\left(p_{x}^{(r)}, q_{x}^{(r)}\right)$ and set $f^{(r)}=\operatorname{dim} P^{(r)}$ $\left(H^{(r)}\right)$. Computing dimensions and signatures, we immediately obtain the following result.

Corollary 2.12. The parameters in Theorem 2.11 are related to the spin dimensions $\left(p_{x}, q_{x}\right)$, the number of space-time points $m$ and the number of 
particles $f$ by

$$
\begin{aligned}
\sum_{r=1}^{R} l_{r} f^{(r)} & =f, \\
p^{(0)}+\sum_{r=1}^{R} l_{r} p^{(r)} & =\sum_{x \in M} p_{x}, \quad q^{(0)}+\sum_{r=1}^{R} l_{r} q^{(r)}=\sum_{x \in M} q_{x}, \\
p_{x}^{(0)}+\sum_{r=1}^{R} l_{r} p_{x}^{(r)} & =p_{x}, \quad q_{x}^{(0)}+\sum_{r=1}^{R} l_{r} q_{x}^{(r)}=q_{x} .
\end{aligned}
$$

\section{A decomposition of $\mathrm{U}(\sigma)$}

We now return to our original problem that the transformation $U$ appearing in the definition of the outer symmetry group (1.2) is not unique due to the gauge freedom (2.1). In order to partially fix the gauge and to characterize the remaining non-uniqueness, in this section we shall bring $U$ in a form compatible with the direct sum decomposition of Theorem 2.11. Before entering the general constructions, we give three simple examples.

Example 3.1. As in Example 2.2, we consider two space-time points with spin dimension $(1,1)$, but now for convenience in the matrix representation

$$
S=\left(\begin{array}{cccc}
1 & 0 & 0 & 0 \\
0 & -1 & 0 & 0 \\
0 & 0 & 1 & 0 \\
0 & 0 & 0 & -1
\end{array}\right), \quad E_{1}=\left(\begin{array}{cc}
\mathbb{1} & 0 \\
0 & 0
\end{array}\right), \quad E_{2}=\left(\begin{array}{cc}
0 & 0 \\
0 & \mathbb{1}
\end{array}\right)
$$

We choose the one-particle fermionic projector $(2.2)$ with $u=2^{-1 / 2}(0,1,0,1)$ and thus

$$
P=\frac{1}{2}\left(\begin{array}{cccc}
0 & 0 & 0 & 0 \\
0 & 1 & 0 & 1 \\
0 & 0 & 0 & 0 \\
0 & 1 & 0 & 1
\end{array}\right)
$$

The free gauge transformations are of the form

$$
U=\operatorname{diag}\left(\mathrm{e}^{\mathrm{i} \alpha}, \mathrm{e}^{\mathrm{i} \varphi}, \mathrm{e}^{\mathrm{i} \beta}, \mathrm{e}^{\mathrm{i} \varphi}\right) \quad \text { with } \alpha, \beta, \varphi \in \mathbb{R},
$$

and thus $\mathcal{F}=U(1) \times U(1) \times U(1)$. When restricting to $P(H)$, this transformation simplifies to $U=\mathrm{e}^{\mathrm{i} \varphi} \mathbb{1}$ and thus $\hat{\mathcal{F}} \simeq U(1)$. Theorem 2.11 gives 
the decomposition

$$
H \simeq H^{(0)} \oplus \mathbb{C} \otimes H^{(1)}
$$

where $\hat{\mathcal{F}}$ acts on the factor $\mathbb{C}$ and

$$
H^{(0)}=\{(a, 0, c, 0): a, c \in \mathbb{C}\}, \quad H^{(1)}=\{(0, b, 0, d): b, d \in \mathbb{C}\}
$$

are both two-dimensional definite subspaces.

Systems (3.1) and (3.2) are symmetric under permutations of the two space-time points. Thus we choose $\mathcal{O}=\{\mathbb{1}, \sigma\}$ with $\sigma$ the transposition of the points 1 and 2 (i.e., $\sigma(1)=2$ and $\sigma(2)=1$ ). The corresponding unitary transformations $U$ as in Definition 1.1 are of the general form

$$
U(\mathbb{1}) \in \mathcal{F}, \quad U(\sigma) \in \mathcal{F} \cdot\left(\begin{array}{ll}
0 & \mathbb{1} \\
\mathbb{1} & 0
\end{array}\right)
$$

The subspace $H^{(0)}$ is trivial in the sense that it is invariant under $E_{1}$ and $E_{2}$, and that $P$ vanishes on it. The fact that $\mathcal{O}$ has a representation on $H^{(0)}$ boils down to the statement that the subspaces $E_{x}\left(H^{(0)}\right)$ have constant signature on the orbits of $\mathcal{O}$. Since this situation is very simple, we do not need to consider $H^{(0)}$ further. Thus, restricting attention to $H^{(1)}$, the transformation $U$ becomes unique up to a phase,

$$
U(\mathbb{1})_{\mid H^{(1)}}=\mathrm{e}^{\mathrm{i} \alpha} \mathbb{1}_{\mid H^{(1)}}, \quad U(\sigma)_{\mid H^{(1)}}=\mathrm{e}^{\mathrm{i} \beta}\left(\begin{array}{cc}
0 & \mathbb{1} \\
\mathbb{1} & 0
\end{array}\right)_{\mid H^{(1)}}
$$

with $\alpha, \beta \in \mathbb{R}$. We want to fix the phases. A first idea is to impose that

$$
\operatorname{det}\left(U(g)_{\mid H^{(1)}}\right)=1 \quad \forall g \in \mathcal{O} .
$$

Unfortunately, as $H^{(1)}$ is two-dimensional, this fixes $U(g)$ only up to a sign. Therefore, it is better to demand that the unitary transformations restricted to $I^{(1)}$ should have determinant one, i.e.,

$$
U(g)_{\mid I^{(1)}} \in \mathrm{SU}\left(I^{(1)}\right) \quad \forall g \in \mathcal{O} .
$$

Then

$$
U^{(1)}(\mathbb{1})=\mathbb{1}_{\mid H^{(1)}}, \quad U^{(1)}(\sigma)=\left(\begin{array}{ll}
0 & \mathbb{1} \\
\mathbb{1} & 0
\end{array}\right)_{\mid H^{(1)}},
$$

giving indeed a representation of the outer symmetry group on $H^{(1)}$. 
Example 3.2. Again in the discrete space-time (3.1), we consider the two-particle fermionic projector

$$
P=\left(\begin{array}{llll}
0 & 0 & 0 & 0 \\
0 & 1 & 0 & 0 \\
0 & 0 & 0 & 0 \\
0 & 0 & 0 & 1
\end{array}\right)
$$

Now the free gauge transformations are of the form

$$
U=\operatorname{diag}\left(\mathrm{e}^{\mathrm{i} \alpha}, \mathrm{e}^{\mathrm{i} \beta}, \mathrm{e}^{\mathrm{i} \gamma}, \mathrm{e}^{\mathrm{i} \delta}\right) \quad \text { with } \alpha, \beta, \gamma, \delta \in \mathbb{R},
$$

and thus $\mathcal{F}=U(1)^{4}$. When restricting to $P(H)$, the factors $\mathrm{e}^{\mathrm{i} \alpha}$ and $\mathrm{e}^{\mathrm{i} \gamma}$ drop out, and thus $\hat{\mathcal{F}} \simeq U(1) \times U(1)$. Theorem 2.11 gives the decomposition

$$
H \simeq H^{(0)} \oplus\left(\mathbb{C} \otimes H^{(1)}\right) \oplus\left(\mathbb{C} \otimes H^{(2)}\right),
$$

where $\hat{\mathcal{F}}$ acts on the factors $\mathbb{C}$ and

$$
H^{(0)}=\{(a, 0, c, 0): a, c \in \mathbb{C}\}, \quad H^{(1)}=\langle(0,1,0,0)\rangle, \quad H^{(2)}=\langle(0,0,0,1)\rangle .
$$

This system is again symmetric under permutations of the two space-time points, $\mathcal{O}=\{\mathbb{1}, \sigma\}$ with $\sigma$ the transposition. The corresponding unitary transformations $U$ as in Definition 1.1 are again of the form (3.3). The subspace $H^{(0)}$ is again trivial. Restricting attention to its complement $H^{(0) \perp}=$ $H^{(1)} \oplus H^{(2)}$, there remains a $U(1) \times U(1)$-freedom,

$$
U(\mathbb{1})_{\mid H^{(0) \perp}}=\left(\begin{array}{cc}
\mathrm{e}^{\mathrm{i} \beta} \mathbb{1} & 0 \\
0 & \mathrm{e}^{\mathrm{i} \delta} \mathbb{1}
\end{array}\right)_{\mid H^{(0) \perp}}, \quad U(\sigma)_{\mid H^{(0) \perp}}=\left(\begin{array}{cc}
0 & \mathrm{e}^{\mathrm{i} \beta} \mathbb{1} \\
\mathrm{e}^{\mathrm{i} \delta} \mathbb{1} & 0
\end{array}\right)_{\mid H^{(0) \perp}} .
$$

In order to fix the phases, we impose that $U$ should be of the form

$$
U_{\mid H^{(0) \perp}}=V\left(U_{1} \oplus U_{2}\right)
$$

with $U_{k} \in \mathrm{SU}\left(I^{(k)}\right)$ and $V$ a permutation matrix, i.e., a $2 \times 2$ matrix with the entries $V_{k^{\prime} k}=\delta_{k^{\prime}, \pi(k)}$, where $\pi \in \mathcal{S}_{2}$ is a permutation. Then $U$ becomes a representation of the outer symmetry group on $H^{(0) \perp}$,

$$
U(\mathbb{1})_{\mid H^{(0) \perp}}=\mathbb{1}_{\mid H^{(0) \perp}}, \quad U(\sigma)_{\mid H^{(0) \perp}}=\left(\begin{array}{ll}
0 & \mathbb{1} \\
\mathbb{1} & 0
\end{array}\right)_{\mid H^{(0) \perp}} .
$$

This example explains why it is, in general, impossible to arrange that the mappings $U$ are invariant on the subspaces $H^{(k)}$. 
Example 3.3. We consider, again in the discrete space-time (3.1), the fermionic projector

$$
P=\left(\begin{array}{cccc}
-\sinh ^{2} \alpha & 0 & 0 & \cosh \alpha \sinh \alpha \\
0 & \cosh ^{2} \alpha & -\cosh \alpha \sinh \alpha & 0 \\
0 & \cosh \alpha \sinh \alpha & -\sinh ^{2} \alpha & 0 \\
-\cosh \alpha \sinh \alpha & 0 & 0 & \cosh ^{2} \alpha
\end{array}\right)
$$

If $\alpha=0$, we are back to Example 3.2. In the case $\alpha \neq 0$, the free gauge formations are all of the form $U=\mathrm{e}^{\mathrm{i} \beta} \mathbb{1}, \beta \in \mathbb{R}$, and thus $\mathcal{F}=U(1)$, and also $\hat{\mathcal{F}}=U(1)$. As a consequence, Theorem 2.11 gives no decomposition, i.e.,

$$
H=H^{(1)} \text {. }
$$

Our system is again permutation-symmetric, $\mathcal{O}=\{\mathbb{1}, \sigma\}$ with $\sigma$ the transposition. The corresponding unitary transformations $U$ as in Definition 1.1 are of the form

$$
U(\mathbb{1})=\mathrm{e}^{\mathrm{i} \beta} \mathbb{1}, \quad U(\sigma)=\mathrm{e}^{\mathrm{i} \gamma}\left(\begin{array}{ll}
0 & \mathbb{1} \\
\mathbb{1} & 0
\end{array}\right)
$$

with $\beta, \gamma \in \mathbb{R}$. In order to fix the phases, we can again prescribe the determinants

$$
\operatorname{det}\left(U(g)_{\mid I^{(1)}}\right):=1 \quad \forall g \in \mathcal{O}
$$

However, since $I^{(1)}$ is two-dimensional, this determines $U(\sigma)$ only up to a sign,

$$
U(\mathbb{1})= \pm \mathbb{1}, \quad U(\sigma)= \pm \mathrm{i}\left(\begin{array}{ll}
0 & \mathbb{1} \\
\mathbb{1} & 0
\end{array}\right)
$$

There seems to be no general method for removing the remaining discrete phase freedom. But in our example, we can clearly fix the phases arbitrarily by setting

$$
U(\mathbb{1})=\mathbb{1}, \quad U(\sigma)=\left(\begin{array}{ll}
0 & \mathbb{1} \\
\mathbb{1} & 0
\end{array}\right)
$$

giving a rsepresentation of the outer symmetry group.

These examples illustrate the following general result. 
Proposition 3.4. In the representation of Theorem 2.11, every unitary transformation $U$ as in Definition 1.1 restricted to $I$ can be represented as

$$
U_{\mid I}=F \cdot W \cdot\left(\bigoplus_{r=1}^{R} \mathbb{1}_{\mathbb{C}^{l} r} \otimes U_{r}\right)
$$

with $F \in \hat{\mathcal{F}}$ and unitary operators $U_{r} \in \mathrm{SU}\left(I^{(r)}\right)$. Here the operator $W$ is a permutation operator in the sense that there is a permutation $\pi \in \mathcal{S}_{R}$ such that for all $u_{r} \in \mathbb{C}^{l_{r}} \otimes I^{(r)}$,

$$
W\left(\bigoplus_{r=1}^{R} u_{r}\right)=\bigoplus_{r=1}^{R} u_{\pi(r)} .
$$

The permutation $\pi$ satisfies the constraints

$$
l_{r}=l_{\pi(r)}, \quad \operatorname{dim} I^{(r)}=\operatorname{dim} I^{(\pi(r))},
$$

and we identify $I^{(r)}$ with $I^{(\pi(r))}$ via an (arbitrarily chosen) isomorphism. For a given choice of these isomorphisms, the operators $W$ are unique, whereas the operators $U_{r}$ are unique up to phase transformations of the form

$$
U_{r} \longrightarrow \mathrm{e}^{\mathrm{i} \vartheta} U_{r} \quad \text { with } \vartheta \cdot \operatorname{dim} I^{(r)} \in 2 \pi \mathbb{Z} .
$$

Proof. For given $\sigma \in \mathcal{O}$ we let $U$ be a unitary transformation satisfying (1.2). Then for every $F \in \mathcal{F}$, the conjugated matrix $F^{U}:=U F U^{-1}$ satisfies the conditions

$$
\begin{aligned}
F^{U} P\left(F^{U}\right)^{-1} & =U F U^{-1} P U F^{-1} U^{-1}=P, \\
F^{U} E_{x}\left(F^{U}\right)^{-1} & =U F U^{-1} E_{x} U F^{-1} U^{-1}=U F E_{\sigma^{-1}(x)} F^{-1} U^{-1} \\
& =U E_{\sigma^{-1}(x)} U^{-1}=E_{x},
\end{aligned}
$$

showing that $F^{U} \in \mathcal{F}$. We write the relation between $F$ and $F^{U}$ in the form

$$
U F=F^{U} U .
$$

According to (2.24), $F$ and $F^{U}$ can be represented as

$$
F_{\mid I}=\bigoplus_{r=1}^{R} F_{r} \otimes \mathbb{1}_{I^{(r)}}, \quad F_{\mid I}^{U}=\bigoplus_{r=1}^{R} F_{r}^{U} \otimes \mathbb{1}_{I^{(r)}} \quad \text { with } F_{r}, F_{r}^{U} \in U\left(l_{r}\right) .
$$

We choose $r, s \in\{1, \ldots, R\}$. Restricting $U$ to $\mathbb{C}^{l_{r}} \otimes I^{(r)}$ and orthogonally projecting its image to $\mathbb{C}^{l_{s}} \otimes I^{(s)}$, we get a mapping

$$
U_{s r}: \mathbb{C}^{l_{r}} \otimes I^{(r)} \longrightarrow \mathbb{C}^{l_{s}} \otimes I^{(s)} .
$$


If this mapping vanishes identically, it can clearly be written in the form

$$
U_{s r}=M_{s r} \otimes A_{s r}
$$

with linear maps

$$
M_{s r}: \mathbb{C}^{l_{r}} \longrightarrow \mathbb{C}^{l_{s}}, \quad A_{s r}: I^{(r)} \longrightarrow I^{(s)} .
$$

Our goal is to show that $U_{s r}$ can also be represented in the form (3.11) and (3.12) if it does not vanish identically. In this case, we define for any non-zero vectors $u^{(r)} \in I^{(r)}$ and $u^{(s)} \in I^{(s)}$ the following injection and projection operators,

$$
\begin{aligned}
& \iota_{r}\left(u^{(r)}\right): \mathbb{C}^{l_{r}} \hookrightarrow I: v \longmapsto v \otimes u^{(r)} \\
& \pi_{s}\left(u^{(s)}\right): I \longrightarrow \mathbb{C}^{l_{s}}: w \longmapsto\left(\left\langle e_{i} \otimes u^{(s)} \mid w\right\rangle\right)_{i=1, \ldots, l_{s}},
\end{aligned}
$$

where $e_{i}$ denotes the canonical basis of $\mathbb{C}^{l_{s}}$. Since $U_{s r}$ is non-trivial, we can choose $u^{(r)}$ such that the product $U \iota_{r}$ is not identically equal to zero. Thus we can choose $u^{(l)}$ such that the operator

$$
M_{s r}:=\pi_{s} U \iota_{r}: \mathbb{C}^{l_{r}} \longrightarrow \mathbb{C}^{l_{s}}
$$

does not vanish identically. Using the representation (3.10) together with (3.9) and the definitions of $\iota_{r}$ and $\pi_{s}$, we obtain for every $F \in \mathcal{F}$,

$$
\pi_{s} U \iota_{r} F_{r}=\pi_{s} U F \iota_{r}=\pi_{s} F^{U} U \iota_{r}=F_{s}^{U} \pi_{s} U \iota_{r},
$$

and thus

$$
M_{s r} F_{r}=F_{s}^{U} M_{s r} \quad \forall F \in \mathcal{F}
$$

Let us show that (3.13) and the fact that $M_{s r} \not \equiv 0$ implies that $M_{s r}$ is bijective: We choose a vector $u \in \mathbb{C}^{l_{r}}$ which is not in the kernel of $M_{s r}$ and set $v=M_{s r} u$. Then for all $F \in \mathcal{F}$,

$$
M_{s r} F_{r} u=F_{s}^{U} v \neq 0,
$$

and since $F_{r} \in U\left(l_{r}\right)$ is arbitrary, it follows that $M_{s r}$ is injective. Moreover, for all $F \in \mathcal{F}$,

$$
F_{s}^{U} v=M_{s r}\left(F_{r} u\right)
$$

and since $F_{s}^{U} \in U\left(l_{s}\right)$ is arbitrary, we see that $M_{s r}$ is surjective. 
The bijectivity of $M_{s r}$ clearly implies that $l_{s}=l_{r}$. Furthermore, we can relate $F_{r}$ and $F_{s}^{U}$ by

$$
F_{s}^{U}=M_{s r} F_{r} M_{s r}^{-1} \text {. }
$$

Restricting both sides of (3.9) to $\mathbb{C}^{l_{r}} \otimes I^{(r)}$ and orthogonally projecting their image to $\mathbb{C}^{l_{s}} \otimes I^{(s)}$, we get the relation

$$
U_{s r}\left(F_{r} \otimes \mathbb{1}_{I^{(r)}}\right)=\left(F_{s}^{U} \otimes \mathbb{1}_{I^{(s)}}\right) U_{s r}
$$

Using (3.14), we obtain

$$
B\left(F_{r} \otimes \mathbb{1}_{I^{(r)}}\right)=\left(F_{r} \otimes \mathbb{1}_{I^{(s)}}\right) B
$$

with $B:=\left(M_{s r}^{-1} \otimes \mathbb{1}_{I^{(s)}}\right) U_{s r}$. Now we can apply Schur's lemma to conclude that $B$ is trivial in its first factor,

$$
\left(M_{s r}^{-1} \otimes \mathbb{1}_{I^{(s)}}\right) U_{s r}=\mathbb{1}_{\mathbb{C}^{l_{r}}} \otimes A_{s r}
$$

for some linear operator $A_{s r}: I^{(r)} \rightarrow I^{(s)}$. Multiplying both sides by $\left(M_{s r} \otimes\right.$ $\left.\mathbb{1}_{I^{(s)}}\right)$ proves the representations (3.11) and (3.12).

Suppose that for a given $r$ there are two $s, s^{\prime} \in\{1, \ldots, R\}$ with $U_{s r} \neq 0 \neq$ $U_{s^{\prime} r}$. Then we obtain from (3.14) that

$$
M_{s r}^{-1} F_{s}^{U} M_{s r}=M_{s^{\prime} r}^{-1} F_{s^{\prime}}^{U} M_{s^{\prime} r} \quad \forall F \in \mathcal{F} .
$$

Since $F_{s}^{U} \in U\left(l_{s}\right)$ can be chosen independently, this relation can hold only if $s=s^{\prime}$. Hence $U_{s r}$ vanishes except for at most one $s$. On the other hand, the surjectivity of $U$ implies that for every $s$ there is at least one $r$ such that $U_{r s} \not \equiv 0$. We conclude that the mapping $r \mapsto s$ is a permutation. We introduce $\pi \in \mathcal{S}_{R}$ such that $s=\pi(r)$. We conclude that

$$
U_{\mid I^{(r)}}=M_{\pi(r) r} \otimes A_{\pi(r) r}: \mathbb{C}^{l_{r}} \otimes I^{(r)} \longrightarrow \mathbb{C}^{l_{\pi(r)}} \otimes I^{(\pi(r))},
$$

and due to the unitarity of $U$, this mapping must be bijective and isometric. In particular, $I^{(r)}$ and $I^{(\pi(r))}$ are isomorphic. Choosing an arbitrary isomorphism $\hat{\kappa}: I^{(r)} \rightarrow I^{(\pi(r))}$, we can write the above mapping as

$$
U_{\mid I^{(r)}}=\left(M_{\pi(r) r} \otimes \mathbb{1}_{I^{(\pi(r))}}\right)\left(\mathbb{1}_{\mathbb{C}^{l_{r}}} \otimes \hat{\kappa}\right)\left(\mathbb{1}_{\mathbb{C}^{l_{r}}} \otimes U_{r}\right)
$$

with $U_{r} \in U\left(I^{(r)}\right)$. For fixed $\hat{\kappa}$, this representation is obviously unique up to the phase transformations

$$
M_{\pi(r) r} \longmapsto \mathrm{e}^{\mathrm{i} \vartheta} M_{\pi(r) r}, \quad U_{r} \longmapsto \mathrm{e}^{-\mathrm{i} \vartheta} U_{r} \quad \text { with } \vartheta \in \mathbb{R} .
$$

These phase transformations can be fixed by imposing that $U_{r} \in \mathrm{SU}\left(I^{(r)}\right)$, except for the discrete phase transformations (3.8). Except for these phases, 
the representation (3.15) is unique, and by restricting (3.6) to $I^{(r)}$, one sees that it coincides precisely with the desired representation of $U_{\mid I}$.

It is useful to write the freedom to perform the phase transformations (3.8) in a group theoretic language. We introduce the abbreviation

$$
f_{r}=\operatorname{dim} I^{(r)},
$$

which is motivated by the fact that $f_{r}$ can be interpreted as the "number of particles in the $r$ th direct summand." The allowed phase factors in (3.8) form a cyclic group of order $f_{r}$, which we denote as usual by

$$
\mathbb{Z}_{f_{r}}=\mathbb{Z} /\left(f_{r} \mathbb{Z}\right)
$$

Multiplying the phase factors by the identity matrix, we regard $\mathbb{Z}_{f_{r}}$ as a normal subgroup of $\mathrm{SU}\left(I^{(r)}\right)$ (it is actually the center of $\mathrm{SU}\left(I^{(r)}\right)$ ). Then $U_{r}$ are uniquely determined as elements of the factor group $\mathrm{SU}\left(I^{(r)}\right) / \mathbb{Z}_{f r}$.

In our next theorem, we extend $U(\sigma)$ from $I$ to $H$.

Theorem 3.5. Let $\left(H,\langle. \mid\rangle,.\left(E_{x}\right)_{x \in M}, P\right)$ be a discrete fermion system with outer symmetry group $\mathcal{O}$. Choosing $H^{(0)}$ maximally $(2.25)$, there are mappings $\lambda^{(r)}: \mathcal{O} \rightarrow U\left(H^{(r)}\right) / \mathbb{Z}_{f_{r}}$ such that for any $\sigma \in \mathcal{O}$ and any choice of representatives $U_{r} \in U\left(H^{(r)}\right)$ of $\lambda^{(r)}(\sigma)$, the resulting unitary operator

$$
U(\sigma)=\mathbb{1}_{H^{(0)}} \oplus W(\sigma) \cdot\left(\bigoplus_{r=1}^{R} \mathbb{1}_{\mathbb{C}^{l_{r}}} \otimes U^{(r)}(\sigma)\right)
$$

satisfies (1.2). The operators $U(\sigma)$ are compatible with the group operations in the sense that for any choice of $U(\sigma)$ and $U(\tau)$, we can choose a representative $U(\sigma \tau)$ such that $U(\sigma) U(\tau)=U(\sigma \tau)$.

Proof. Let us choose a convenient basis in every subspace $H_{x}^{(r)}:=E_{x}^{(r)}$ $\left(H^{(r)}\right), x \in M, r \in\{1, \ldots, R\}$. We closely follow the construction of the special basis of $H_{x}$ in the Proof of Lemma 2.8. First, in every subspace $I_{x}^{(r)}:=E_{x}^{(r)} P^{(r)}\left(H^{(r)}\right)$ we choose a non-degenerate subspace of maximal dimension and in this subspace a pseudo-orthonormal basis $\left(e_{i}^{(x, r)}\right)$. We extend this basis by vectors $f_{j}^{(x, r)}$ to a basis of $I_{x}^{(r)}$. Next we choose vectors $h_{j}^{(x, r)} \in$ $H_{x}^{(r)}$ which are conjugate to the $f_{j}^{(x, r)}$ in the sense that $\left\langle f_{i}^{(x, r)} \mid h_{j}^{(x, r)}\right\rangle=\delta_{i j}$. Then $\left(e_{i}^{(x, r)}, f_{j}^{(x, r)}, h_{j}^{(x, r)}\right)$ is a basis of $H_{x}^{(r)}$, as the following argument shows. Suppose that $v \in H_{x}^{(r)}$ is a vector in the orthogonal complement of the span of $\left(e_{i}^{(x, r)}, f_{j}^{(x, r)}, h_{j}^{(x, r)}\right)$. Then the vector space $\mathbb{C}^{l_{r}} \otimes\{v\} \subset H$ is orthogonal to $I$ and thus in the kernel of $P$. Furthermore, it is invariant under the 
projectors $E_{x}$. Using that $H^{(0)}$ is maximal (2.25), we conclude that $\mathbb{C}^{l_{r}} \otimes$ $\{v\} \subset H^{(0)}$ and thus $v=0$.

For any $\sigma \in \mathcal{O}$ we choose a $\tilde{U}$ satisfying (1.2). Then $\left.\tilde{U}\right|_{I}$ is of the form (3.6). Multiplying $\tilde{U}$ by a suitable free gauge transformation, we can arrange that

$$
\left.\tilde{U}\right|_{I}=\bigoplus_{r=1}^{R} \mathbb{1}_{\mathbb{C}^{l_{r}}} \otimes U_{r}
$$

with $U_{r} \in U\left(I^{(r)}\right)$. Our task is to extend the operators $U_{r}$ to $H^{(r)}$. To this end, we first note that

$\tilde{U}\left(I_{x}^{(r)}\right)=\left(\tilde{U} E_{x} P\right)\left(H^{(r)}\right)=\left(E_{\sigma(x)} P \tilde{U}\right)\left(H^{(r)}\right)=\left(E_{\sigma(x)} P\right)\left(H^{(\pi(r))}\right)=I_{\sigma(x)}^{(\pi(r))}$,

showing that $\tilde{U}$ maps $I_{x}^{(r)}$ to $I_{\sigma(x)}^{(\pi(r))}$. Since $\tilde{U}$ is unitary, this mapping is clearly isometric and bijective. Introducing the isomorphism $\kappa$ in (3.15) by mapping the basis vectors $\left(e_{i}^{(x, r)}, f_{j}^{(x, r)}, h_{j}^{(x, r)}\right)$ to the corresponding basis vectors $\left(e_{i}^{(\sigma(x), \pi(r))}, f_{j}^{(\sigma(x), \pi(r))}, h_{j}^{(\sigma(x), \pi(r))}\right)$, the mapping $U_{r} \in U\left(I^{(r)}\right)$ in (3.15) is locally unitary according to Definition 2.6. Thus we can apply Lemma 2.8 to unitarily extend $U_{r}$ to $H^{(r)}$. More precisely, we choose the extension on the subspaces $H_{x}^{(r)}$ according to (2.16). The resulting mapping $\lambda^{(r)}$ : $U\left(I^{(r)}\right) \rightarrow U\left(H^{(r)}\right)$ allows us to define $U(\sigma)$ by

$$
U(\sigma)_{\mid H^{(r)}}=\left(\mathbb{1}_{\mathbb{C}^{l_{r}}} \otimes \kappa\right)\left(\mathbb{1}_{\mathbb{C}^{l_{r}}} \otimes \lambda\left(U_{r}\right)\right) .
$$

This formula depends on our particular choice of $\kappa$. But we can modify it so as to be valid for a general isomorphism $\hat{\kappa}: I^{(r)} \rightarrow I^{(\pi(r))}$. To this end, we simply rewrite $U(\sigma)$ as

$$
\begin{gathered}
U(\sigma)_{\mid H^{(r)}}=\left(\mathbb{1}_{\mathbb{C}^{l_{r}}} \otimes \hat{\kappa}\right)\left(\mathbb{1}_{\mathbb{C}^{l_{r}}} \otimes \hat{\lambda}\left(U_{r}\right)\right) \\
\text { with } \hat{\lambda}(\sigma):=(\hat{\kappa})^{-1} \circ \kappa(\sigma) \circ \lambda .
\end{gathered}
$$

Comparing with (3.15) and choosing $U(\sigma)_{\mid H^{(0)}}=\mathbb{1}_{H^{(0)}}$, we can write $U(\sigma)$ in the form (3.16) (where clearly each $U_{r}$ in (3.16) is identified with the corresponding operator $\hat{\lambda}\left(U_{r}\right)$ in $\left.(3.18)\right)$.

Let us analyze the arbitrariness of the above construction. The operators $U_{r} \in \mathrm{SU}\left(I^{(r)}\right)$ in (3.17) are unique up to the discrete phase transformations (3.8). Since $\hat{\lambda}$ is linear, we find that their extensions $U_{r} \in U\left(H^{(r)}\right)$ in (3.16) are also unique up to discrete phases. Hence the functions $\lambda^{(r)}$ in the statement of the theorem are indeed well-defined mappings from $\mathcal{O}$ to $U\left(H^{(r)}\right) / \mathbb{Z}_{f r}$. Conversely, different representatives of $\lambda^{(r)}(\sigma)$ differ only by discrete phase transformations (3.7). According to Proposition 3.4, such transformations do not affect (1.2). 
Finally, we need to verify that $U(\sigma)$ is compatible with the group operations: Since the $U_{r}$ in Proposition 3.4 are unique up to the discrete phase transformations (3.7), it is obvious that the restrictions $u(\sigma)_{\mid I}$ are compatible with the group operations. Furthermore, as we fixed the bases $\left(e_{i}^{(x, r)}, f_{j}^{(x, r)}\right.$, $\left.h_{j}^{(x, r)}\right)$ and extended the $U_{r}$ simply by mapping corresponding basis vectors onto each other and by using the explicit formula (2.16), we conclude that the extensions $U(\sigma)$ are also compatible with the group operations.

The just-constructed isomorphisms $I^{(r)} \simeq I^{(\pi(r))}$ and $H_{x}^{(r)} \simeq H_{\sigma(x)}^{(\pi(r))}$ immediately imply the following relations between dimensions and signatures.

Corollary 3.6. Assume that in the representation of Theorem 2.11, the vector space $H^{(0)}$ is chosen maximally (2.25). Then the parameters in Theorem 2.11 and Proposition 3.4 are related to each other for all $x \in M$ and $r \in\{1, \ldots, R\}$ by

$$
\begin{aligned}
f^{(r)} & =f^{(\pi(r))}, \\
\left(p^{(r)}, q^{(r)}\right) & =\left(p^{(\pi(r))}, q^{(\pi(r))}\right), \\
\left(p_{x}^{(r)}, q_{x}^{(r)}\right) & =\left(p_{\sigma(x)}^{(\pi(r))}, q_{\sigma(x)}^{(\pi(r))}\right) .
\end{aligned}
$$

It is important to observe that the unitary transformations $U_{r}$ in Proposition 3.4 and Theorem 3.5 can be arbitrarily changed by the phase transformations (3.8). This so-called discrete phase freedom is undesirable, because as a consequence the mapping $\sigma \mapsto U(\sigma)$ with $U(\sigma)$ as in (3.16) is not uniquely defined and, in particular, is not a group representation. In special situations (see Example 3.3 and Proposition 9.4), one can fix the phases to obtain a representation of the outer symmetry group. However, there seems to be no general method for fixing the phases. This difficulty can be understood from the following analogy to the continuum theory: Minkowski space is symmetric under Lorentz transformations; thus we can regard $\mathrm{SO}(1,3)$ as an outer symmetry group. In this setting, the vectors of $H$ should correspond to Dirac wave functions. In order to represent the outer symmetry group, one would have to find a representation of $\mathrm{SO}(1,3)$ on the Dirac spinors. However, such a representation does not exist in non-mathematical terms, because a spatial rotation by $360^{\circ}$ flips the sign of the spinors. The way out is to extend the outer symmetry group by going over to the universal cover $\operatorname{Spin}(1,3)$ of $\mathrm{SO}(1,3)$. The spin group then has a unitary representation on $H$.

In the discrete setting, the situation is more involved than in the continuum, because in Proposition 3.4 and Theorem 3.5 the phase freedom 
depends on the number of direct summands $R$ and on the number of particles $f_{r}$ in each direct summand. For this reason, our method is to first decompose our discrete fermion system into smaller subsystems (Section 4). For each of the resulting subsystems, we then treat the discrete phase freedom similarly as in the continuum by extending the outer symmetry group (Section 5).

\section{Simple systems and simple subsystems}

In this section, we want to decompose a given discrete fermion system with outer symmetry group $\mathcal{O}$ into subsystems which should be as small as possible. These smaller subsystems can easily be identified in the direct sum decompositions of Proposition 3.4 and Theorem 3.5: On the space $H^{(0)}$, the fermionic projector vanishes, and therefore we call $\left(H^{(0)},\left(E_{x}^{(0)}\right)_{x \in M}, P=0\right)$ a trivial system. Next, we consider the group of all permutation operators $W(\mathcal{O}) \subset S_{R}$. If the group elements act as permutations on $\{1, \ldots, R\}$, the corresponding orbits give a partition of $\{1, \ldots, R\}$ into disjoint subsets. It is an important observation that the direct summands corresponding to different orbits are never mapped into each other in (3.16). Thus for any orbit $Q \subset\{1, \ldots, R\}$, the subsystem

$$
\hat{H}=\bigoplus_{r \in Q} \mathbb{C}^{l} \otimes H^{(r)}
$$

with

$$
\hat{E}_{x}=\bigoplus_{r \in Q} \mathbb{1}_{\mathbb{C}^{l}} \otimes E_{x}^{(r)}, \quad \hat{P}=\bigoplus_{r \in Q} \mathbb{1}_{\mathbb{C}^{l}} \otimes P^{(r)}
$$

is again a discrete fermion system with outer symmetry group $\mathcal{O}$. Note that the parameter $l_{r}$ is constant on the orbits (3.7), and thus we could simply set $l=l_{r}$.

Systems (4.1) and (4.2) can be decomposed further, because they consist of $l$ identical copies of the discrete fermion system

$$
\tilde{H}=\bigoplus_{r \in Q} H^{(r)}, \quad \tilde{E}_{x}=\bigoplus_{r \in Q} E_{x}^{(r)}, \quad \tilde{P}=\bigoplus_{r \in Q} P^{(r)}
$$

which again has outer symmetry $\mathcal{O}$. We refer (4.3) as a simple system. To go one step further, we can also consider one direct summand of (4.3), i.e., 
for any $r_{0} \in Q$ the system

$$
\left(H^{\left(r_{0}\right)},\left(E_{x}^{\left(r_{0}\right)}\right)_{x \in M}, P^{\left(r_{0}\right)}\right) .
$$

This system does not have outer symmetry $\mathcal{O}$. But we can introduce

$$
\mathcal{N}:=\left\{\sigma \in \mathcal{O} \text { with } \sigma\left(r_{0}\right)=r_{0}\right\}
$$

as the outer symmetry group if for any $\sigma \in \mathcal{N}$ we take $U(\sigma)=\left.U_{r}\right|_{H^{\left(r_{0}\right)}}$ : $H^{\left(r_{0}\right)} \rightarrow H^{\left(r_{0}\right)}$. We call (4.4) together with the outer symmetry group (4.5) a simple subsystem. As we shall see, using the coset structure of $\mathcal{N} \subset \mathcal{O}$ we can completely reconstruct the corresponding simple system from a simple subsystem.

We now introduce the above subsystems without referring to our original discrete fermion system. This has the advantage that they can later be used as "building blocks" for constructing general discrete fermion systems. We always keep the discrete space-time points $M=\{1, \ldots, m\}$ as well as the outer symmetry group $\mathcal{O} \subset \mathcal{S}_{m}$ fixed.

Definition 4.1. Let $\left(H,\langle. \mid\rangle,.\left(E_{x}\right)_{x \in M}, P\right)$ be a discrete space-time. Assume that the spin dimension is constant on the orbits of $\mathcal{O}$,

$$
p_{x}=p_{\sigma(x)}, \quad q_{x}=q_{\sigma(x)} \quad \forall x \in M, \forall \sigma \in \mathcal{O} .
$$

We set $P=0$. Then the system $\left(H,\langle. \mid\rangle,.\left(E_{x}\right)_{x \in M}, P\right)$ is called a trivial system.

For a trivial system, $\mathcal{O}$ can be realized as an outer symmetry group. Namely, since the spin dimension is constant on the orbits of $\mathcal{O}$, we can choose pseudo-orthonormal bases of the subspaces $E_{x}(H)$ and identify the corresponding basis vectors to obtain isomorphisms between $E_{x}(H)$ and $E_{\sigma(x)}(H)$. Using these isomorphisms, one immediately gets the unitary transformation $U$ satisfying (2.1).

Definition 4.2. Let $\left(H,\langle. \mid\rangle,.\left(E_{x}\right)_{x \in M}, P\right)$ be a discrete space-time. Assume that we are given a subgroup $\mathcal{N} \subset \mathcal{O}$ together with a unitary representation $U$ of $\mathcal{N}$ on $H$. Assume furthermore that the following conditions are satisfied:

(i) $\mathcal{N}$ is an outer symmetry group (see Definition 2.1).

(ii) The system contains no trivial subsystems, i.e.,

$$
J \subset H \text { definite, } \quad P(J)=0 \text { and } \quad E_{x}(J) \subset J \forall x \in M \Longrightarrow J=\varnothing .
$$

(iii) The proper free gauge group is simply the $U(1)$ of global phase transformations,

$$
\hat{\mathcal{F}}=\left\{\mathrm{e}^{\mathrm{i} \vartheta} \mathbb{1} \text { with } \vartheta \in \mathbb{R}\right\}
$$


Then the structure $\left(H,\langle. \mid\rangle,.\left(E_{x}\right)_{x \in M}, P, \mathcal{N} \subset \mathcal{O}\right)$ is called a simple subsystem.

We denote the number of particles of a simple subsystem by $f_{\text {sub }}:=$ $\operatorname{rank}(P)$.

Let us construct the corresponding simple system. We denote the cosets $\{\sigma \mathcal{N}$ with $\sigma \in \mathcal{O}\}$ by $C_{1}, \ldots, C_{K}$; they form a partition of the set $\mathcal{O}$. Of each coset we choose one representative $\sigma_{k} \in C_{k}$. For convenience, we set $C_{1}=\mathcal{N}$ and choose $\sigma_{1}=\mathbb{1}$. Every $\sigma \in \mathcal{N}$ defines via $\tau \mathcal{N} \mapsto(\sigma \tau) \mathcal{N}$ a permutation of the cosets $C_{1}, \ldots, C_{K}$. This yields a homomorphism from $\mathcal{O}$ to the symmetric group $\mathcal{S}_{K}$, which we denote by $\pi$,

$$
\pi: \mathcal{O} \longrightarrow \mathcal{S}_{K}
$$

Clearly, $\left(\pi\left(\sigma_{k}\right)\right)(1)=k$, and thus the subgroup $\pi(\mathcal{O}) \subset \mathcal{S}_{K}$ acts transitively on the set $\{1, \ldots, K\}$.

We introduce the inner product space $\tilde{H}=\mathbb{C}^{K} \otimes H$ (with the natural inner product $(2.19))$. On $\tilde{H}$ we introduce the projectors $\tilde{P}$ and $\tilde{E}_{x}$ by

$$
\begin{aligned}
\left.\tilde{P}\right|_{\left\langle e_{k}\right\rangle \otimes H} & =\left.(\mathbb{1} \otimes P)\right|_{\left\langle e_{k}\right\rangle \otimes H}, \\
\left.\tilde{E}_{\sigma_{k}(x)}\right|_{\left\langle e_{k}\right\rangle \otimes H} & =\left.\left(\mathbb{1} \otimes E_{x}\right)\right|_{\left\langle e_{k}\right\rangle \otimes H},
\end{aligned}
$$

where $\left(e_{k}\right)$ denotes the canonical basis of $\mathbb{C}^{K}$. Furthermore, we introduce for all $k, l \in\{1, \ldots K\}$ the canonical identification maps

$$
\kappa_{l, k}:\left\langle e_{k}\right\rangle \otimes H \subset \tilde{H} \longrightarrow\left\langle e_{l}\right\rangle \otimes H: e_{k} \otimes u \longmapsto e_{l} \otimes u .
$$

In order to define the unitary operators $\tilde{U}$ on $\tilde{H}$, we introduce for any $\sigma \in \mathcal{O}$ and $k \in\{1, \ldots, K\}$ the parameter $l=(\pi(\sigma))(k)$. Then the group element $\tau:=\sigma_{l}^{-1} \sigma \sigma_{k}$ satisfies the condition

$$
(\pi(\tau))(1)=\left(\pi\left(\sigma_{l}^{-1} \sigma \sigma_{k}\right)\right)(1)=\left(\pi\left(\sigma_{l}^{-1} \sigma\right)\right)(k)=\left(\pi\left(\sigma_{l}^{-1}\right)\right)(l)=1,
$$

and thus $\tau \in \mathcal{N}$. Hence we may define $\tilde{U}(\sigma)$ by

$$
\left.\tilde{U}(\sigma)\right|_{\left\langle e_{k}\right\rangle \otimes H}=\left.\kappa_{l, k} \circ(\mathbb{1} \otimes U(\tau))\right|_{\left\langle e_{k}\right\rangle \otimes H} .
$$

Lemma 4.3. The discrete fermion system $\left(\tilde{H},\left(\tilde{E}_{x}\right)_{x \in M}, \tilde{P}\right)$ has the outer symmetry group $\mathcal{O}$, with the corresponding unitary operators $\tilde{U}$ as given by (4.9). The definitions (4.7)-(4.9) are, up to isomorphisms, independent of the choice of the group elements $\sigma_{k} \in \mathcal{O}$. The number of particles $f:=\operatorname{rank} \tilde{P}$ is given by

$$
f=f_{\text {sub }} \frac{\# \mathcal{O}}{\# \mathcal{N}}
$$


Proof. By taking the trace of (4.7), one sees that $f=k f_{\text {sub }}$. Since the number of cosets is clearly given by $k=\# \mathcal{O} / \# \mathcal{N}$, we obtain (4.10).

We only consider the transformation of the space-time projectors $\tilde{E}_{x}$, because the fermionic projector transforms in exactly the same way, except for the simplification that it does not carry a space-time index. For any $\sigma \in \mathcal{O}$ and $k \in\{1, \ldots, K\}$, we set $l=(\pi(\sigma))(k)$ and $\tau=\sigma_{l}^{-1} \sigma \sigma_{k}$. Then, setting $x=\sigma_{k}^{-1} y$, we have for all $y \in M$,

$$
\begin{aligned}
\left.\tilde{U}(\sigma) \tilde{E}_{y} \tilde{U}(\sigma)^{-1}\right|_{\left\langle e_{l}\right\rangle \otimes H} & =\left.(\mathbb{1} \otimes U(\tau))\left(\mathbb{1} \otimes E_{x}\right)(\mathbb{1} \otimes U(\tau))^{-1}\right|_{\left\langle e_{l}\right\rangle \otimes H} \\
& =\left.\left(\mathbb{1} \otimes E_{\tau(x)}\right)\right|_{\left\langle e_{l}\right\rangle \otimes H}=\tilde{E}_{\left(\sigma_{l} \circ \tau\right)(x)}=\tilde{E}_{\sigma(y)},
\end{aligned}
$$

where in the last line we used that $\tau \in \mathcal{N}$ and that $\mathcal{N}$ is a symmetry of the simple subsystem represented by $U$.

Suppose that $\bar{\sigma}_{k} \in \mathcal{O}$ is another choice of group elements with $\left(\pi\left(\bar{\sigma}_{k}\right)\right)$ $(1)=k$. Then

$$
\left(\pi\left(\bar{\sigma}_{k}^{-1} \sigma_{k}\right)\right)(1)=\left(\pi\left(\bar{\sigma}_{k}\right)^{-1} \circ \pi\left(\sigma_{k}\right)\right)(1)=\left(\pi\left(\bar{\sigma}_{k}\right)^{-1}\right)(k)=1,
$$

and thus $\tau_{k}:=\bar{\sigma}_{k}^{-1} \sigma_{k} \in \mathcal{N}$. Using that $\mathcal{N}$ is an outer symmetry group of the simple subsystem, we find

$$
\begin{aligned}
\left.\tilde{\bar{E}}_{x}\right|_{\left\langle e_{k}\right\rangle \otimes H} & =\left.\left(\mathbb{1} \otimes E_{\bar{\sigma}_{k}^{-1}(x)}\right)\right|_{\left\langle e_{k}\right\rangle \otimes H}=\left.\left(\mathbb{1} \otimes E_{\left(\tau_{k} \circ \sigma_{k}^{-1}\right)(x)}\right)\right|_{\left\langle e_{k}\right\rangle \otimes H} \\
& =\left.\left(\mathbb{1} \otimes U\left(\tau_{k}\right) E_{\sigma_{k}^{-1}(x)} U\left(\tau_{k}\right)^{-1}\right)\right|_{\left\langle e_{k}\right\rangle \otimes H},
\end{aligned}
$$

showing that the objects defined using $\sigma_{k}$ and those defined using $\bar{\sigma}_{k}$ are related to each other by the unitary transformation $V$ given by

$$
\left.V\right|_{\left\langle e_{k}\right\rangle \otimes H}=\left.\left(\mathbb{1} \otimes U\left(\tau_{k}\right)\right)\right|_{\left\langle e_{k}\right\rangle \otimes H} .
$$

Hence our definitions are unique up to isomorphisms.

Definition 4.4. We call the system $\left(\tilde{H},\langle. \mid\rangle,.\left(\tilde{E}_{x}\right)_{x \in M}, \tilde{P}, \mathcal{O}, \tilde{U}\right)$ the simple system corresponding to the simple subsystem of Definition 4.2.

Theorem 4.5. Let $\left(H,\langle. \mid\rangle,.\left(E_{x}\right)_{x \in M}, P\right)$ be a discrete fermion system with outer symmetry group $\mathcal{O}$. Then there is a trivial system $\left(H^{(0)},\left(E_{x}^{(0)}\right)_{x \in M}\right.$, $\left.P^{(0)}\right)$ as well as a collection of simple systems $\left(\tilde{H}^{(a)},\left(\tilde{E}_{x}^{(a)}\right)_{x \in M}, \tilde{P}^{(a)}\right.$, $\left.K_{a}\right)_{a=1, \ldots, A}, A \geq 1$, together with parameters $n_{a} \in \mathbb{N}$ such that we have the 
following isomorphisms:

$$
\begin{aligned}
H & \simeq H^{(0)} \oplus\left(\bigoplus_{a=1}^{A} \mathbb{C}^{n_{a}} \otimes \tilde{H}^{(a)}\right), \\
E_{x} & \simeq E_{x}^{(0)} \oplus\left(\bigoplus_{a=1}^{A} \mathbb{1}_{\mathbb{C}^{n_{a}}} \otimes \tilde{E}_{x}^{(a)}\right), \\
P & \simeq \bigoplus_{a=1}^{A} \mathbb{1}_{\mathbb{C}^{n_{a}}} \otimes \tilde{P}^{(a)} .
\end{aligned}
$$

Proof. We present the discrete fermion system as in Theorem 2.11 with $H^{(0)}$ maximal (2.25) and represent the outer symmetry as in Proposition 3.4 and Theorem 3.5. The orbits of the permutation matrices $W(\sigma)$ form a partition of the set $\{1, \ldots, R\}$. Let $Q \subset\{1, \ldots, R\}$ be one of these orbits. By reordering the space-time points, we can arrange that $Q=\{1, \ldots, K\}$ with a parameter $K$ in the range $1 \leq K \leq R$. From (3.7) and Corollary 3.6, we know that

$$
\bigoplus_{r \in Q} \mathbb{C}^{l_{r}} \otimes H^{(r)}=\mathbb{C}^{l_{1}} \otimes \mathbb{C}^{K} \otimes H^{(1)}
$$

The action of $W(\sigma)$ on $Q$ defines a transitive group homomorphism $\pi: \mathcal{O} \rightarrow$ $P_{K}$. We introduce the subsets $C_{1}, \ldots, C_{K}$ by

$$
C_{k}=\{\sigma \in \mathcal{O} \mid(\pi(\sigma))(1)=k\} .
$$

Clearly, $\mathcal{N}:=C_{1}$ is a subgroup of $\mathcal{O}$. Let us verify that $C_{k}$ coincides with the cosets of $\mathcal{N}$ in $\mathcal{O}$ : For $\sigma, \tau \in C_{k}$, the calculation

$$
\left(\pi\left(\sigma^{-1} \tau\right)\right)(1)=\left(\pi(\sigma)^{-1} \circ \pi(\tau)\right)(1)=\left(\pi(\sigma)^{-1}\right)(k)=1
$$

shows that $\sigma^{-1} \tau \in \mathcal{N}$, and thus $\sigma$ and $\tau$ belong to the same coset. If conversely $\sigma$ and $\tau$ are in the same coset, we know that $\sigma^{-1} \tau \in \mathcal{N}$ and thus $\left(\pi(\sigma)^{-1} \pi(\sigma)\right)(1)=1$. In other words,

$$
(\pi(\sigma))(1)=(\pi(\tau))(1)=: k,
$$

meaning that $\sigma, \tau \in C_{k}$.

Identifying the $C_{k}$ with the cosets of $\mathcal{N}$, the above homomorphism coincides precisely with the action of $\mathcal{O}$ on the cosets as described by (4.6). According to (2.22) and (2.23), the projectors $P$ and $E_{x}$ act only on the last factor in the decomposition (4.14) and can thus be regarded as operators on $H^{(1)}$. For the resulting subsystem $\left(H^{(1)}, P, E_{x}\right), \mathcal{N}$ is an outer symmetry group. The maximality condition (2.25) implies condition (ii) in Definition 4.2, whereas condition (iii) in Definition 4.2 follows from the representation of the proper free gauge group (2.24). We conclude that $\left(H^{(1)}, E_{x}, P, \mathcal{N}\right)$ is a simple subsystem. 
Let $\left(\tilde{H}^{(1)}, \tilde{E}_{x}, \tilde{P}\right)$ be the corresponding simple system. Then the tensor product of $\mathbb{C}^{l_{1}}$ with this system is obviously isomorphic to the restriction of our original system to the subspace (4.14). Taking the direct sum of $H^{(0)}$ with the so obtained systems corresponding to the different orbits of $V$ yields our original discrete fermion system.

Example 4.6. Let us build up the discrete fermion systems considered in Examples 3.1-3.3. In both examples (3.2) and (3.4), we obtain the trivial system by restricting the fermion system to the subspace

$$
H^{(0)}=\{(a, 0, c, 0): a, c \in \mathbb{C}\}
$$

In the example (3.2), the simple subsystem is constructed as follows. We set $H=\mathbb{C}^{2}$ with $-\langle. \mid$.$\rangle equal to the canonical scalar product on \mathbb{C}^{2}$ and introduce the projectors

$$
E_{1}=\left(\begin{array}{ll}
1 & 0 \\
0 & 0
\end{array}\right), \quad E_{2}=\left(\begin{array}{ll}
0 & 0 \\
0 & 1
\end{array}\right), \quad P=\frac{1}{2}\left(\begin{array}{ll}
1 & 1 \\
1 & 1
\end{array}\right) .
$$

We again let $\mathcal{O}=\{\mathbb{1}, \sigma\}$ with $\sigma$ the transposition. We choose $\mathcal{N}=\mathcal{O}$ with the representation

$$
U(\mathbb{1})=\mathbb{1}, \quad U(\sigma)=\left(\begin{array}{ll}
0 & 1 \\
1 & 0
\end{array}\right)
$$

Then there is only one coset, $K=1$. Furthermore, $\pi$ is the trivial mapping $\pi(\sigma)=\mathbb{1}$. This system is a simple subsystem according to Definition 4.2. Since $K=1$, this subsystem coincides with the corresponding simple system. Obviously, the direct sum of this system with $H^{(0)}$ is isomorphic to systems (3.1) and (3.2).

In the example (3.4), we construct the simple subsystem by choosing $H=\mathbb{C}$ with $\langle u \mid v\rangle=-\bar{u} v$. Furthermore, we choose

$$
E_{1}=1, \quad E_{2}=0, \quad P=1 .
$$

We again let $\mathcal{O}=\{\mathbb{1}, \sigma\}$ with $\sigma$ the transposition. But now we choose $\mathcal{N}=\{\mathbb{1}\}$ equal to the trivial subgroup. Then its representation is also trivial, $U(\mathbb{1})=\mathbb{1}$. This system satisfies all the conditions in Definition 4.2. There are two cosets of $\mathcal{N}$ in $\mathcal{O}, K=2$. Hence the corresponding simple system lives in the inner product space $\tilde{H}=\mathbb{C}^{2} \times H \simeq \mathbb{C}^{2}$, where $-\langle. \mid$.$\rangle coincides$ with the canonical scalar product on $\mathbb{C}^{2}$. The resulting representation $\tilde{U}$ given by (4.9) coincides with (4.15). A short calculation using (4.7) and 
(4.8) yields

$$
E_{1}=\left(\begin{array}{ll}
1 & 0 \\
0 & 0
\end{array}\right), \quad E_{2}=\left(\begin{array}{cc}
0 & 0 \\
0 & 1
\end{array}\right) \quad P=\left(\begin{array}{ll}
1 & 0 \\
0 & 1
\end{array}\right) .
$$

This is a simple fermion system with outer symmetry group $\mathcal{O}$ consisting of two simple subsystems. Taking the direct sum with the trivial system $H^{(0)}$ gives precisely the systems (3.1) and (3.4).

In Example 3.3, the discrete fermion system with $P$ according to (3.5) is a simple subsystem with $\mathcal{N}=\mathcal{O}$. Thus it cannot be decomposed into smaller components.

We finally give a useful characterization of simple systems which does not refer to simple subsystems.

Proposition 4.7. Let $\left(\tilde{H},\langle. \mid\rangle,.\left(\tilde{E}_{x}\right)_{x \in M}, \tilde{P}, \mathcal{O}\right)$ be a discrete fermion system with outer symmetry group $\mathcal{O}$. This system can be realized as a simple fermion system according to Definition 4.4 if and only if the following two conditions are satisfied:

(a) The system contains no trivial subsystems according to condition (ii) in Definition 4.2 .

(b) The system cannot be decomposed into the direct sum of two non-trivial fermion systems $\left(H^{1}, E_{x}^{1}, P^{1}\right)$ and $\left(H^{2}, E_{x}^{2}, P^{2}\right)$,

$$
\tilde{H}=H^{1} \oplus H^{2}, \quad \tilde{E}_{x}=E_{x}^{1} \oplus E_{x}^{2}, \quad \tilde{P}=P^{1} \oplus P^{2},
$$

which both have the outer symmetry group $\mathcal{O}$.

Proof. It is obvious from condition (ii) in Definition 4.2 and our above construction that a simple system contains no trivial subsystems. Furthermore, a simple subsystem cannot be decomposed into non-trivial subsystems because otherwise the proper free gauge group would contain independent phase transformations of both subsystems and thus $\hat{\mathcal{F}} \supset U(1) \times U(1)$, in contradiction to condition (iii) in Definition 4.2. The corresponding simple system is, by construction, the smallest system with outer symmetry group $\mathcal{O}$ which contains the simple subsystem, and therefore it cannot be decomposed into smaller systems with these properties.

Assume conversely that a discrete fermion system $\left(\tilde{H},\langle. \mid\rangle,.\left(\tilde{E}_{x}\right)_{x \in M}, \tilde{P}, \mathcal{O}\right)$ satisfies the assumptions stated in the proposition. We again present the discrete fermion system as in Theorem 2.11 with $H^{(0)}$ maximal (2.25) and represent the unitary transformations as in Proposition 3.4 and Theorem 3.5. Then the assumption (a) implies that $H^{(0)}$ is trivial. Furthermore, the 
group $\pi(\mathcal{O}) \subset \mathcal{S}_{R}$ must act transitively on the set $\{1, \ldots, R\}$ because otherwise the orbits of $\pi(\mathcal{O})$ would give a splitting of the fermion system into non-trivial smaller systems with outer symmetry group $\mathcal{O}$, in contradiction to assumption (b). Hence there is only one orbit $Q=\{1, \ldots, R\}$, and the construction in the Proof of Theorem 4.5 shows how the system $\left(\tilde{H},\langle. \mid\rangle,.\left(\tilde{E}_{x}\right)_{x \in M}, \tilde{P}, \mathcal{O}\right)$ is realized as the simple system corresponding to a suitable simple subsystem.

\section{A representation of a group extension of $\mathcal{N}$}

In this section, we shall construct a unitary representation of the outer symmetry. The method is to remove the discrete phase freedom by extending the outer symmetry group. For convenience, we restrict attention to our smallest building block: the simple subsystem (see Definition 4.2). We first specialize the result of Theorem 3.5 to a simple subsystem.

Corollary 5.1. Let $\left(H,\langle. \mid\rangle,.\left(E_{x}\right)_{x \in M}, P, \mathcal{N} \subset \mathcal{O}\right)$ be a simple subsystem. Then there is an injective group homomorphism

$$
\lambda: \mathcal{N} \hookrightarrow \mathrm{U}(H) / \mathbb{Z}_{f_{\text {sub }}}
$$

such that for all $\sigma \in \mathcal{N}$ and any representative $U \in U(H)$ of $\lambda(\sigma)$ the symmetry relation (1.2) holds.

Proof. We regard the simple subsystem as a discrete fermion system with outer symmetry group $\mathcal{N}$ and decompose it according to Proposition 3.4 and Theorem 3.5. Since there are no trivial subsystems (see condition (ii) in Definition 4.2), we know that $H^{(0)}=\{0\}$. From the fact that $\hat{\mathcal{F}}=U(1)$ (see condition (iii) in Definition 4.2) we conclude furthermore that $R=1$ and that $l_{r}=1$. Hence

$$
H \simeq H^{(1)}, \quad P \simeq P^{(1)}, \quad E_{x} \simeq E_{x}^{(1)} .
$$

Moreover, the permutation operator $W$ in (3.16) is trivial. Thus there is a homomorphism $\lambda^{(1)}: \mathcal{N} \rightarrow U(H) / \mathbb{Z}_{f_{\text {sub }}}$ such that any representative $U=$ $U^{(1)}$ of $\lambda(\sigma):=\lambda^{(1)}(\sigma)$ satisfies $(1.2)$.

It remains to show that $\lambda$ is injective. For any $\sigma, \sigma^{\prime} \in \mathcal{N}$ with $\sigma \neq \sigma^{\prime}$ there is a space-time point $x \in M$ such that $\sigma(x) \neq \sigma^{\prime}(x)$. Then any representative $U(\sigma)$ of $\lambda(\sigma)$ maps $E_{x}(H)$ to $E_{\sigma(x)}(H)$, whereas $U\left(\sigma^{\prime}\right)$ maps $E_{x}(H)$ to $E_{\sigma^{\prime}(x)}(H)$. Thus obviously $U(\sigma) \neq U\left(\sigma^{\prime}\right)$ and also $\lambda(\sigma) \neq \lambda\left(\sigma^{\prime}\right)$. 
We now form the set of unitary matrices

$$
\hat{\mathcal{N}}=\{U \in U(H) \mid U \text { represents } \lambda(\sigma) \text { with } \sigma \in \mathcal{N}\} .
$$

This is a discrete subgroup of $U(H)$, because $\lambda$ is a homomorphism. This group has a natural action on $M$ defined by $U x=\sigma(x)$ if $U$ represents $\lambda(\sigma)$. We consider $\hat{\mathcal{N}}$ as an abstract group, whereas the identification with the particular $U \in U(H)$ is denoted by $\hat{\lambda}$. The subset

$$
\{U \in H \mid U \text { represents } \lambda(\mathbb{1})\} \subset \hat{\mathcal{N}}
$$

is an abelian subgroup of $\hat{\mathcal{N}}$, which can be identified with $\mathbb{Z}_{f_{\text {sub }}}$. This subgroup is normal in $\hat{\mathcal{N}}$ and clearly $\hat{\mathcal{N}} / \mathbb{Z}_{f_{\text {sub }}}=\mathcal{N}$. The result of this construction is summarized as follows.

Theorem 5.2. There is a central extension $\hat{\mathcal{N}}$ of $\mathcal{N}$ by $\mathbb{Z}_{f_{\text {sub }}}$ together with a faithful group representation $\hat{\lambda}: \hat{\mathcal{N}} \rightarrow U(H)$ such that the following commutative diagram is exact:

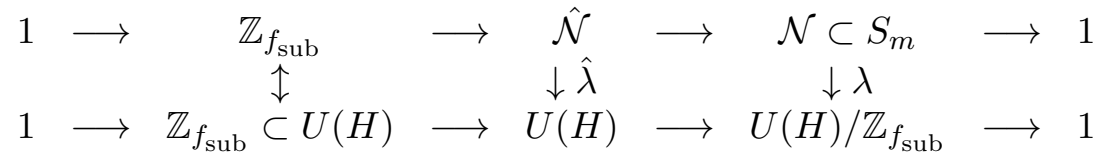

(where 1 is the trivial group). If $\hat{\mathcal{N}}$ is equipped with the natural action on $M$ inherited from $\mathcal{N}$, the mapping $\hat{\lambda}$ represents the outer symmetry in the sense that

$$
U P U^{-1}=P, \quad U E_{x} U^{-1}=E_{\sigma(x)} \quad \forall x \in M, \forall \sigma \in \hat{\mathcal{N}},
$$

where $U=\hat{\lambda}(\sigma)$.

The above situation simplifies if $\hat{\mathcal{N}}$ possesses a subgroup $\mathcal{J}$ which still is a group extension of $\mathcal{N}$. In this case, we obtain by restriction of $\hat{\lambda}$ the exact commutative diagram

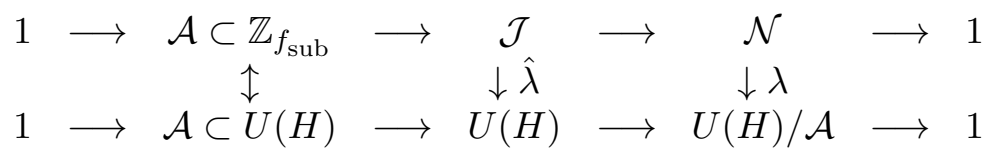

where $\mathcal{A}$ is a suitable subgroup of $\mathbb{Z}_{f_{\text {sub }}}$. This has the advantage that the group $\mathcal{J}$ has fewer elements than $\hat{\mathcal{N}}$, making it easier to construct its representation $\hat{\lambda}$. In the simplest case when $\hat{\mathcal{N}}$ is a product,

$$
\hat{\mathcal{N}}=\mathbb{Z}_{f_{\text {sub }}} \times \mathcal{N}
$$

we can choose $\mathcal{J}=\mathcal{N}$ and $\mathcal{A}=1$. With a slight abuse of notation, in what follows we shall in the situation (5.2) denote $\mathcal{J}$ by $\hat{\mathcal{N}}$. Thus $\hat{\mathcal{N}}$ always denotes a central extension of $\mathcal{N}$ having a unitary representation of on $H$; 
the group $\hat{\mathcal{N}}$ is either the group in Theorem 5.2 or a suitable subgroup of this group.

Having a unitary representation of $\hat{\mathcal{N}}$ is very useful because it allows us to decompose a simple subsystem into irreducible components.

Proposition 5.3. Let $\left(H,\langle. \mid\rangle,.\left(E_{x}\right)_{x \in M}, P, \mathcal{N} \subset \mathcal{O}\right)$ be a simple subsystem and $\hat{\mathcal{N}}$ a central extension of $\mathcal{N}$ together with a unitary representation $U$ of $\hat{\mathcal{N}}$ satisfying (5.1). Then there are inequivalent irreducible representations $\left(R_{l}, \mathbb{C}^{d_{l}}\right)_{l=1, \ldots, L}$ of $\hat{\mathcal{N}}$ such that $H$ has an orthogonal decomposition of the form

$$
H \simeq \bigoplus_{l=1}^{L} \mathbb{C}^{d_{l}} \otimes H^{[l]}
$$

where $H^{[l]}$ are inner product spaces of signature $\left(p^{[l]}, q^{[l]}\right)$. The unitary representation of $\hat{\mathcal{N}}$ and the fermionic projector take the form

$$
U(\sigma) \simeq \bigoplus_{l=1}^{L} R_{l}(\sigma) \otimes \mathbb{1}_{H^{[l]}}, \quad P \simeq \bigoplus_{l=1}^{L} \mathbb{1}_{\mathbb{C}^{d_{l}}} \otimes P^{[l]},
$$

where $P^{[l]}$ are projectors in $H^{[l]}$ with negative-definite image.

Proof. The proposition follows immediately from Lemma 2.10 and Schur's lemma.

\section{The pinned symmetry group}

In Theorem 5.2, we constructed a finite group $\hat{\mathcal{N}}$ acting on $M$ together with a unitary representation $U$ of $\mathcal{N}$ on an inner product space $(H,\langle. \mid\rangle$.$) . This$ representation satisfies, for all $\sigma \in \hat{\mathcal{N}}$ and $x \in M$, the condition

$$
U(\sigma) E_{x} U(\sigma)^{-1}=E_{\sigma(x)}
$$

plus the symmetry condition for the fermionic projector $U P U^{-1}=P$. In this section, we disregard the symmetry condition for the fermionic projector and consider unitary representations of $\hat{\mathcal{N}}$ which only satisfy (6.1). Our goal was to use the gauge freedom to bring such representations into a simple form.

Because of the completeness of the space-time projectors, we can consider instead of $U(\sigma)$ the operator products $E_{x} U(\sigma) E_{y}$ for $x, y \in M$. We denote

the orbits of the action of $\hat{\mathcal{N}}$ on $M$ by $M_{1}, \ldots, M_{J}, J \geq 1$. The orbits form a partition of $M$, and we can introduce an equivalence relation $x \simeq y$ 
by identifying the points on the same orbit. Rewriting (6.1) as $U(\sigma) E_{y}=$ $E_{\sigma(y)} U(\sigma)$ and multiplying from the left by $E_{x}$, we find that

$$
E_{x} U(\sigma) E_{y}=0 \quad \text { unless } x \simeq y .
$$

Therefore, it suffices to consider the case that $x$ and $y$ are on the same orbit. Without loss of generality, we can assume that $x, y \in M_{1}$. In other words, it remains to consider the following restriction of $U$,

$$
U_{\mid H_{1}} \quad \text { with } H_{1}:=\bigoplus_{x \in M_{1}} E_{x}(H) .
$$

Furthermore, there is no loss of generality to distinguish one point of $M_{1}$, because this point can be mapped to any other point of $M_{1}$ by applying $\hat{\mathcal{N}}$. For simplicity, we assume that $1 \in M_{1}$. We now form the subgroup of the outer symmetry group which leaves this distinguished point invariant.

Definition 6.1. The pinned symmetry group $\mathcal{R} \subset \hat{\mathcal{N}}$ is the group of all $\sigma \in \hat{\mathcal{N}}$ with $\sigma(1)=1$.

For every $\sigma \in \mathcal{R}$, we find that $U(\sigma) E_{1}=E_{\sigma(1)} U(\sigma)=E_{1} U(\sigma)$. In other words, $U(\sigma)$ maps the subspace $\tilde{H}:=E_{1}(H)$ into itself. Hence

$$
V(\sigma):=U(\sigma)_{\mid \tilde{H}} \text { is a unitary representation of } \mathcal{R} \text { on } \tilde{H} \text {. }
$$

The next proposition gives a procedure to reconstruct $U_{\mid H_{1}}$ from a given representation $V$.

Proposition 6.2. Let $\left(H,\langle. \mid\rangle,.\left(E_{x}\right)_{x \in M}\right)$ be a discrete space-time. Assume that we are given a group $\hat{\mathcal{N}}$ acting on $M$ such that the spin dimension is constant on the orbits of $\hat{\mathcal{N}}$. Let $M_{1} \subset M$ be the orbit containing the point $1 \in M$. Suppose that $V$ is a unitary representation of the corresponding pinned symmetry group $\mathcal{R}$ (see Definition 6.1 ) on $\tilde{H}:=E_{1}(H)$. Then there is, up to gauge transformations, a unique unitary representation $U$ of $\hat{\mathcal{N}}$ on $H_{1}$ (see (6.3)) which satisfies for all $x \in M_{1}$ the conditions (6.1) and which, when restricted to $\mathcal{R}$ and $\tilde{H}$, coincides with $V$.

Proof. Since $\hat{\mathcal{N}}$ acts transitively on $M_{1}$, we can for every $x \in M_{1}$ choose a group element $\sigma_{x} \in \hat{\mathcal{N}}$ with the property that $\sigma_{x}(1)=x$. For convenience, we choose $\sigma_{1}=\mathbb{1}$. Since the spin dimension is, by assumption, constant on the orbits of $\hat{\mathcal{N}}$, the spaces $E_{x}(H), x \in M_{1}$, are all isomorphic. Thus for every $x \in M_{1}$, we can choose an isomorphism $\kappa_{x}: \tilde{H} \rightarrow E_{x}(H)$. For convenience, we choose $\kappa_{1}=\mathbb{1}$. We define $U\left(\sigma_{x}\right)$ restricted to $\tilde{H}$ by

$$
U\left(\sigma_{x}\right)_{\mid \tilde{H}}: \tilde{H} \longrightarrow E_{x}(H): u \longmapsto \kappa_{x}(u) .
$$

Together with the given representation of $\mathcal{R}$ on $\tilde{H}$, (6.5) uniquely determines a representation of $\hat{\mathcal{N}}$ on $H$. Namely, suppose that for a given $\sigma \in$ 
$\hat{\mathcal{N}}$ and $x \in M_{1}$, we want to construct $U(\sigma)_{\mid E_{x}(H)}$. Setting $y=\sigma(x)$, we rewrite $\sigma$ in the form $\sigma=\sigma_{y} \rho \sigma_{x}^{-1}$. Then $\rho$ is an element of $\mathcal{R}$ and using that $U$ should be a group representation

$$
U(\sigma)_{\mid E_{x}(H)}=U\left(\sigma_{y}\right)_{\mid \tilde{H}} V(\rho)_{\mid \tilde{H}} U\left(\sigma_{x}\right)_{\mid E_{x}(H)}^{-1} .
$$

All the operators on the right-hand side are given. It is straightforward to verify that the operators (6.6) form a representation of $\hat{\mathcal{N}}$ on $H$ satisfying (6.1).

For the uniqueness question, we let $U$ be any unitary representation of $\hat{\mathcal{N}}$ on $H$ satisfying (6.1). Then for all $x \in M_{1}$, the operator $U\left(\sigma_{x}\right)_{\mid \tilde{H}}$ is a unitary operator from $\tilde{H}$ to $E_{x}(H)$. By a local gauge transformation at $x$, we can arrange that this operator coincides with $\kappa_{x}$. Thus we can achieve by a suitable gauge transformation that $U$ satisfies the conditions (6.5). But then $U$ is uniquely determined according to (6.6).

\section{Building up general systems: a constructive procedure}

The constructions of the previous sections yield a systematic procedure for constructing all discrete fermion systems for a given outer symmetry group $\mathcal{O}$ and for given values of the parameters $\left(p_{x}, q_{x}\right), m$ and $f$. We denote the maximal spin dimension by $n=\max _{x \in M}\left\{p_{x}, q_{x}\right\}$.

1. Choose a subgroup $\mathcal{N}$ of $\mathcal{O}$. Choose a parameter $f_{\text {sub }}$ with $1 \leq f_{\text {sub }} \leq$ $f \cdot \# \mathcal{N} / \# \mathcal{O}$.

2. Consider a central extension $\hat{\mathcal{N}}$ of $\mathcal{N}$ by $\mathbb{Z}_{f_{\text {sub }}}$,

$$
0 \longrightarrow \mathbb{Z}_{f_{\text {sub }}} \longrightarrow \hat{\mathcal{N}} \longrightarrow \mathcal{N} \longrightarrow 0 .
$$

If $\hat{\mathcal{N}}$ has a subgroup, which is also a central extension of $\mathcal{N}$, one may replace $\hat{\mathcal{N}}$ by this subgroup (see Section 6 ).

3. Determine the orbits $M_{1}, \ldots M_{J}, J>0$, of the action of $\hat{\mathcal{N}}$ on $M$.

4. Choose in every orbit one representative $x_{j} \in M_{j}$ and determine the corresponding pinned symmetry groups $\mathcal{R}_{j}$ (see Definition 6.1).

5. Choose a unitary representation $V$ of each pinned symmetry group on a corresponding indefinite inner product space $\hat{H}_{j}$ of signature $\left(p_{j}, q_{j}\right)$ and $p_{j}, q_{j} \leq n$. The irreducible subspaces of this representation can be chosen to be definite (see Lemma 2.10). The dimensions of the irreducible subspaces must be at most $n$.

6. The construction of Proposition 6.2 gives a unitary representation $U$ of $\hat{\mathcal{N}}$ on a discrete space-time $\left(H,\langle. \mid\rangle,.\left(E_{x}\right)_{x \in M}\right)$ satisfying $(6.1)$. 
7. After completely reducing the obtained representation $U$ on each of the invariant subspaces

$$
H_{j}:=\bigoplus_{x \in M_{j}} E_{x}(H)
$$

one can characterize all projectors $P$ which satisfy the condition $U P U^{-1}=P$ (see Proposition 5.3). We build up projectors $P$ onto negative-definite subspaces of dimension $f_{\text {sub }}$.

8. Selecting those projectors $P$ which satisfy the conditions (ii) and (iii) in Definition 4.2, we obtain simple subsystems. Carrying out the constructions (4.7)-(4.9) yield corresponding simple systems (see Definition 4.4), whose number of particles is given by $f_{\text {sub }} \# \mathcal{O} / \# \mathcal{N}$.

9. According to Theorem 4.5, a general discrete fermion system is obtained from simple systems by taking tensor products with $\mathbb{C}^{k}$ and by taking direct sums. We must satisfy the conditions that the spin dimension of the resulting system must nowhere exceed $(n, n)$ and that the total number of particles should be equal to $f$.

\section{Examples: abelian outer symmetries and lattices}

We now illustrate the construction steps of the previous section in a few examples. For simplicity, we consider only the case $\hat{\mathcal{N}}=\mathcal{N}$ of a trivial central extension.

Definition 8.1. A discrete fermion system $\left(H,\langle. \mid\rangle,.\left(E_{x}\right)_{x \in M}, P\right)$ with outer symmetry group $\mathcal{O}$ is said to be homogeneous if $\mathcal{O}$ acts transitively on $M$.

Example 8.2. (Homogeneous systems with abelian outer symmetry) Let us consider the case of a homogeneous discrete fermion system with abelian outer symmetry group. Then $\mathcal{O}$ acts transitively on $M$, and thus for every $x \in M$ we can choose a group element $\sigma_{x} \in \mathcal{O}$ with $\sigma_{x}(1)=x$. The corresponding pinned symmetry group $\mathcal{R}$ is trivial, because for every $\sigma \in \mathcal{R}$,

$$
\sigma(x)=\left(\sigma_{x} \sigma \sigma_{x}^{-1}\right)(x)=\left(\sigma_{x} \sigma\right)(1)=\sigma_{x}(1)=x \quad \forall x \in M,
$$

and thus $\sigma=\mathbb{1}$. As a consequence, for every $x \in M$ the choice of $\sigma_{x}$ is unique. In particular, the order $\# \mathcal{O}$ of the symmetry group equals the number $m$ of space-time points, and we can use the mapping $x \mapsto \sigma_{x}$ to identify $M$ with $\mathcal{O}$.

According to the basis theorem (see [4, Chapter II, Section 10]), every finite abelian group is the direct sum of cyclic groups of prime power order. Thus there are parameters $\left(l_{n}\right)_{n=1, \ldots, N}$, each being a power of a prime $p_{n}$, and corresponding group elements $g_{n}$ with the properties that the $\left(g_{n}\right)_{n=1, \ldots, N}$ 
generate $\mathcal{O}$ and that each of the groups $\left\{g_{n}^{k}: k \in \mathbb{Z}\right\}$ is cyclic of order $l_{n}$. Introducing the group $\mathcal{T}=l_{1} \mathbb{Z} \oplus \cdots \oplus l_{N} \mathbb{Z}$, we can write $\mathcal{O}$ as the quotient group

$$
\mathcal{O}=\mathbb{Z}^{N} / \mathcal{T}
$$

Identifying the points $x \in M$ with the corresponding group elements $\sigma_{x} \in \mathcal{O}$, we can regard $M$ as an $N$-dimensional lattice with side lengths $l_{n}$.

Let $(\hat{H},\langle. \mid\rangle$.$) be an indefinite inner product space of signature (p, q)$. Since $\mathcal{R}$ is trivial, its only representation on $\hat{H}$ is $V \equiv \mathbb{1}$. The construction of Proposition 6.2 yields that the corresponding discrete space-time $\left(H,\langle. \mid\rangle,.\left(E_{x}\right)_{x \in M}\right)$ and the representation $U$ of the outer symmetry group $\mathcal{O}$ can be given as follows:

$$
\begin{gathered}
H=\mathbb{C}^{M} \otimes \hat{H}, \quad M=\mathbb{Z}^{N} / \mathcal{T}, \\
E_{x}: e_{y} \otimes u \longmapsto \delta_{x y} e_{y} \otimes u, \\
U(\sigma): e_{y} \otimes u \longmapsto e_{\sigma(y)} \otimes u .
\end{gathered}
$$

In other words, $H$ consists of $\hat{H}$-valued functions on $M$, and $U$ acts on these functions by translating the points of $M$ by the action of the group $\mathcal{O}$. It is convenient to use the short notation

$$
u(x)=E_{x} u \in\left\langle e_{x}\right\rangle \otimes \hat{H} \simeq \hat{H},
$$

where in the last step we identify the vector spaces in the natural way.

In order to completely reduce $U$, we first note that, since $\mathcal{O}$ is abelian, its irreducible representations are all one-dimensional. Thus our task is to decompose $H$ into one-dimensional subspaces which are invariant under the action of $U$. An easy calculation shows that the subspaces spanned by the vectors

$$
u(x)=\hat{u} \exp \left(i \sum_{n=1}^{N} k_{n} x_{n}\right)
$$

with

$$
\hat{u} \in \hat{H}, \quad k_{n} \in\left\{0,1 \frac{2 \pi}{l_{n}}, 2 \frac{2 \pi}{l_{n}}, \ldots,\left(l_{n}-1\right) \frac{2 \pi}{l_{n}}\right\}
$$

are invariant under the action of $U$. Also, while counting dimensions one sees that these vectors form a basis of $H$, and therefore the subspaces spanned by the vectors (8.1) completely reduce $U$. The fermionic projectors which satisfy the conditions $U P U^{-1}=P$ must be invariant on the irreducible 
subspaces, and this means that they must be of the form

$$
P=\sum_{x, y \in M} \sum_{k \in \mathcal{K}} \kappa_{x, y} P^{(k)} E_{y} \exp \left(i \sum_{n=1}^{N} k_{n}\left(x_{n}-y_{n}\right)\right),
$$

where $\mathcal{K}$ is a set of vectors $k=\left(k_{n}\right)_{n=1, \ldots, N}$ with components in the range as in (8.2). Here $P^{(k)}$ are projectors on negative-definite subspaces in $\hat{H}$ and $\kappa_{x, y}$ is the natural isomorphism from $E_{y}(H)$ to $E_{x}(H)$.

Clearly, the vectors (8.1) are plane waves on the lattice $M$ with periodic boundary conditions, and (8.3) is the general form of a projector which is "diagonal in momentum space." We conclude that the construction procedure of Section 7 reduces to the usual discrete Fourier transform on a finite lattice, with the only difference that the side lengths $l_{n}$ are always prime powers.

Example 8.3. (General systems with abelian outer symmetry) As in the previous example, we consider an abelian group $\mathcal{O}$, but which now does not necessarily act transitively on $M$. We denote the orbits of the action of $\mathcal{O}$ on $M$ by $M_{1}, \ldots, M_{L}$. We let $\mathcal{K}_{l}$ be the subgroups of $\mathcal{O}$ which keep the sets $M_{l}$ fixed. Since every subgroup of an abelian group is normal, we can form the quotient groups $\mathcal{O}_{l}=\mathcal{O} / \mathcal{K}_{l}$. Then the groups $\mathcal{O}_{l}$ can be regarded as a group of permutations on the sets $M_{l}$, which act transitively. Therefore, on each of the orbits $M_{l}$ we can use the method of Example 8.2 to construct a discrete "sub-space-time" $\left(H_{l},\left(E_{x}\right)_{x \in M_{l}}\right)$ together with a unitary representation $U_{l}$ of the outer symmetry group $\mathcal{O}_{l}$. Since a representation of an outer symmetry is trivial between different orbits (6.2), the discrete space-time is obtained simply by taking the direct sums of the sub-space-times.

In order to construct the fermionic projector, we first note that the irreducible subspaces of $H$ are precisely the span of the plane waves (8.1) of all the sub-space-times. Let $\kappa$ be an irreducible representation of $\mathcal{O}$. We form the subspace $H_{\kappa} \subset H$ spanned by all those invariant subspaces on which $U$ is equivalent to $\kappa$. According to Lemma $2.10, H_{\kappa}$ is a non-degenerate subspace of $H$. The most general fermionic projector satisfying the symmetry condition $U P U^{-1}=P$ is the operator which is invariant on the subspaces $H_{\kappa}$ corresponding to the different irreducible representations of $\mathcal{O}$ and is on each of these subspaces a projector on a negative-definite subspace (see Proposition 5.3).

Example 8.4. (Two-dimensional lattice with pinned symmetry) To give an example with a non-trivial pinned symmetry group, we next consider a discrete space-time which, similar to Example 8.2, is a finite lattice, but now with a larger, non-abelian symmetry group. For a given prime power 
$l>2$, we introduce the group $\mathcal{T}=l \mathbb{Z} \oplus l \mathbb{Z}$ as well as the square lattice

$$
M=\mathbb{Z}^{2} / \mathcal{T}
$$

We let $\mathcal{S}$ be the group of all isometries of $\mathbb{R}^{2}$ which map the lattice points $\mathbb{Z}^{2} \subset \mathbb{R}^{2}$ onto themselves (thus $\mathcal{S}$ is the group of all translations, reflections and rotations about multiples of the angle $90^{\circ}$ ). A short consideration shows that $\mathcal{T}$ is a normal subgroup of $\mathcal{S}$. We let $\mathcal{O}$ be the corresponding quotient group

$$
\mathcal{O}=\mathcal{S} / \mathcal{T}
$$

This group has a natural action on $M$ which corresponds to translations, reflections and rotations on a square lattice whose opposite sides are identified.

Since $\mathcal{O}$ contains the translations, which act transitively on $M$, our system is clearly homogeneous. Thus we can arbitrarily distinguish one point of $M$; for convenience, we denote the origin in $\mathbb{Z}^{2} / \mathcal{T}$ by 1 . To construct the corresponding pinned symmetry group, we introduce the two unitary matrices

$$
\alpha=\left(\begin{array}{cc}
0 & -1 \\
1 & 0
\end{array}\right), \quad \rho=\left(\begin{array}{cc}
1 & 0 \\
0 & -1
\end{array}\right) .
$$

These matrices describe a rotation by $90^{\circ}$ and the reflection at the $x_{2}$-axis of $\mathbb{R}^{2}$, respectively. Since they are compatible with the lattice structure of $\mathbb{Z}^{2}$ and the action of $\mathcal{T}$, they can be regarded as elements of $\mathcal{O}$. Furthermore, they leave the origin of $\mathbb{Z}^{2}$ fixed, and thus $\alpha, \rho \in \mathcal{R}$. Since by composing $90^{\circ}$ rotations with reflections we obtain all elements of the pinned symmetry group, it is obvious that $\mathcal{R}$ is generated by $\alpha$ and $\rho$. Note that $\alpha$ and $\rho$ do not commute and thus $\mathcal{R}$ is non-abelian.

The next step is to construct a representation $V$ of $\mathcal{R}$ on an indefinite inner product space $(\hat{H},\langle. \mid\rangle$.$) . The possibilities depend on the signature (p, q)$ of $\hat{H}$. One possible choice clearly is the trivial representation

$$
V(\alpha)=\mathbb{1}=V(\beta) .
$$

Another possibility is to choose the sign representation

$$
V(\alpha)=\mathbb{1}, \quad V(\beta)=-\mathbb{1} .
$$

If $p>1$ or $q>1$, more complicated representations are possible. For example, one can take direct sums of the one-dimensional representations (8.5) and (8.6). In this case, the corresponding representation $U$ of $\mathcal{O}$ will also split into a direct sum of representations corresponding to the irreducible summands of $V$, and therefore this case is straightforward. Moreover, one can choose higher-dimensional irreducible representations of $\mathcal{R}$. To give a 
simple example, we consider the two-dimensional irreducible representation by the matrices in (8.4),

$$
V(\alpha)=\left(\begin{array}{cc}
0 & -1 \\
1 & 0
\end{array}\right), \quad V(\rho)=\left(\begin{array}{cc}
1 & 0 \\
0 & -1
\end{array}\right) .
$$

Let us construct the corresponding representations $U$ on $H$. For every $x \in M$, we choose the unique translation $\sigma_{x} \in \mathcal{O}$ with $\sigma_{x}(1)=x$. Carrying out the construction of Proposition 6.2 for the trivial representation (8.5), we obtain $H=\mathbb{C}^{M} \otimes \hat{H}$ and

$$
E_{x}: e_{y} \otimes u \longmapsto \delta_{x y} e_{y} \otimes u, \quad U(\sigma): e_{y} \otimes u \longmapsto e_{\sigma(y)} \otimes u .
$$

In the case of the sign representation (8.6), we obtain the same discrete space-time as for the trivial representation, with the only difference that the resulting representation $U$ also involves signs,

$$
U(\sigma): e_{y} \otimes u \longmapsto \operatorname{sgn}(\sigma) e_{\sigma(y)} \otimes u,
$$

where $\operatorname{sgn}(\sigma)$ equals -1 if $\sigma$ changes the orientation and equals +1 otherwise. In the case of the two-dimensional irreducible representation (8.7), we obtain the same discrete space-time as for the trivial representation, but now with $\hat{H}=\mathbb{C}^{2}$ and the resulting representation $U$ given by

$$
U(\sigma): e_{y} \otimes u \longmapsto e_{\sigma(y)} \otimes V(\sigma)(u),
$$

where in order to define $V(\sigma)$ we compose $\sigma$ by a translation in order to arrange that the origin is fixed. The resulting group element is in the pinned symmetry group, and taking its representation matrix $V$ defines us $V(\sigma)$.

It remains to completely reduce $U$. To this end, we first note that for the subgroup of translations, $U$ coincides precisely with the representation $U$ in Example 8.2. Thus the invariant subspaces of this subgroup are again the plane waves $\phi_{k, \hat{u}}$ of the form

$$
\phi_{k, \hat{u}}(x)=\hat{u} \exp (\mathrm{i}\langle k, x\rangle)
$$

with $\hat{u} \in \hat{H}$ and $\langle.,$.$\rangle the canonical scalar product on \mathbb{R}^{2}$. Here the momentum vector $k=\left(k_{1}, k_{2}\right)$ must be in the "dual lattice" $\mathcal{K}$,

$$
k \in \mathcal{K}:=\left\{0,1 \frac{2 \pi}{l}, 2 \frac{2 \pi}{l}, \ldots,(l-1) \frac{2 \pi}{l}\right\}^{2} .
$$

In order to get the invariant subspaces of the whole group $\mathcal{O}$, we form the subspaces of plane wave solutions which are mapped into each other by the action of $\mathcal{R}$,

$$
H_{k}:=\left\{\phi_{\rho(k), \hat{u}} \mid \rho \in \mathcal{R}, \hat{u} \in \hat{H}\right\} \subset H,
$$

where $\rho(k)$ is the action of $\mathcal{R}$ induced on the dual-lattice via the relation $\langle k, x\rangle=\langle\rho(k), \rho(x)\rangle$. If $k=0$, the dimension of $H_{k}$ coincides with the 
dimension $d$ of $\hat{H}$ (i.e., it is equal to 1 if $V$ is the trivial or sign representation, and it equals 2 for the representation (8.7)). In the cases $k_{1}=0, k_{2}=0$ or $k_{1}=k_{2}$ (and $\left.k=\left(k_{1}, k_{2}\right) \neq 0\right), H_{k}$ is of dimension $4 d$. In the remaining case $0 \neq k_{1} \neq k_{2} \neq 0$, the orbit of $\mathcal{R}$ on $k$ consists of eight points and thus $\operatorname{dim} H_{k}=8 d$. On these low-dimensional subspaces, $U$ can be completely reduced in a straightforward way; we leave the details to the reader.

\section{Spontaneous breaking of the permutation symmetry}

In this section, we consider discrete fermion systems whose outer symmetry group $\mathcal{O}$ is the symmetric group $\mathcal{S}_{m}$ of all permutations of the space-time points. Such systems are clearly homogeneous (see Definition 8.1). This implies that the spaces $E_{x}(H)$ must all be isomorphic, and thus the spin dimension is constant in space-time,

$$
\left(p_{x}, q_{x}\right)=(n, n) \quad \forall x \in M
$$

We first give a physical motivation of our main result. If a physical system is modeled by a discrete fermion system, the parameter $n$ is known (e.g., $n=2$ for the simplest system involving Dirac spinors [3]), whereas the number $m$ of space-time points will be very large. The number $f$ of particles will also be very large, but much smaller than the number of space-time points (note that we also count the states of the Dirac sea as being occupied by particles, see $[1,3]$, and as these states lie on a three-dimensional surface in fourdimensional momentum space, their number scales typically like $f \sim m^{3 / 4}$ ). Hence the case of physical interest is

$$
n \ll f \ll m .
$$

Our next theorem will show that in this case no discrete fermion systems with outer symmetry group $\mathcal{S}_{m}$ exist. In other words, the permutation symmetry of discrete space-time is necessarily destroyed by the fermionic projector, and thus a spontaneous symmetry breaking occurs. Our result can be understood in non-technical terms as follows: One possibility to build up fermion systems with permutation symmetry is to take fermions which are "spread out" over all of space-time. The orthogonality of the fermionic states implies that the number of such states can be at most as large as the spin dimension. Hence not all the particles can be "completely delocalized" in this way. Another method is to "localize" the particles at individual space-time points. But then the permutation symmetry implies that there must be a particle at every space-time point, and the number of particles will be as large as $m$, which is impossible. The next theorem makes the above consideration precise and rules out all other ways of building in the fermions. 
Theorem 9.1. Suppose that $\left(H,\langle. \mid\rangle,.\left(E_{x}\right)_{x \in M}, P\right)$ is a discrete fermion system of spin dimension $(n, n)$. Assume that the number of space-time points $m$ is sufficiently large, then

$$
m>\left\{\begin{array}{cl}
3 & \text { if } n=1, \\
\max \left(2 n+1,4\left[\log _{2} n\right]+6\right) & \text { if } n>1
\end{array}\right.
$$

(where $[x]=\max \{k \in \mathbb{Z}, k \leq x\}$ is the Gauß bracket) and that the number of particles $f$ lies in the range

$$
n<f<m-1
$$

Then the discrete fermion system cannot have the outer symmetry group $\mathcal{O}=\mathcal{S}_{m}$.

The remainder of the paper is devoted to the proof of this theorem. The symmetric group has two obvious one-dimensional representations: the trivial representation $U(\sigma)=\mathbb{1}$ and the sign representation $U(\sigma)=\operatorname{sgn}(\sigma) \mathbb{1}$. Lemma 9.2 gives a lower bound for the dimensions of all other irreducible representations.

Lemma 9.2. Let $U$ be an irreducible representation of $\mathcal{S}_{k}$ on $\mathbb{C}^{N}$, which is neither the trivial nor the sign representation. Then

$$
N \geq \frac{k}{2}
$$

Proof. The representation theory for the symmetric group is formulated conveniently using Young diagrams (for a good introduction see, e.g. [6, Section 2.8]). Every irreducible representation of $\mathcal{S}_{k}$ corresponds to a Young diagram with $k$ positions. The Young diagram $\lambda$ corresponding to $U$ has more than one row (otherwise $U$ would be the trivial representation) and more than one column (otherwise $U$ would be the sign representation). The hook formula (see [6, Section 2.8 and Appendix C.5]) states that the dimension $N$ of the representation is given by

$$
N=\frac{k !}{\prod(\text { all hook lengths in } \lambda)},
$$

where the hook length of any position in a Young diagram is defined as the sum of positions to its right plus the number of positions below it plus one.

We consider the subdiagram $\mu$ of all the positions consisting of the last column having more than one position plus all the positions to its right. In 
the following example, the subdiagram $\mu$ is marked by stars:

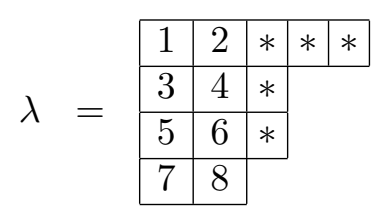

We denote the number of positions of $\mu$ by $l$ and the number of its rows by $r$. Obviously, $l \geq r \geq 2$. We compute the hook lengths of all positions of $\mu$ and substitute them in (9.3) as

$$
N=\frac{k !}{l(r-1) !(l-r) !} \frac{1}{\prod(\text { all hook lengths not in } \mu)} .
$$

When computing the hook length of any position which is not in $\mu$, at most $(l-r+1)$ of the "stared squares" of $\mu$ contribute (because at most the stared squares in one row are counted). Furthermore, ordering the positions of $\lambda \backslash \mu$ beginning from the upper-left corner as indicated in the figure, one can arrange that the hook length of any position does not involve all the previous positions. Hence the hook length of the first position is at most $(k-l)+(l-r+1)=k-r+1$, the hook length of the second position is at most $k-r$, and so on. We conclude that

$$
\begin{aligned}
N & \geq \frac{k !}{l(r-1) !(l-r) !} \frac{1}{(k-r+1)(k-r) \cdots(l-r+2)} \\
& =\frac{k !(l-r+1)}{l(r-1) !(k-r+1) !}=\frac{l-r+1}{l}\left(\begin{array}{c}
k \\
r-1
\end{array}\right) .
\end{aligned}
$$

We consider two cases. If $k=l$, the diagrams $\lambda$ and $\mu$ coincide, and since our Young diagram has more than one column, we know that $k>r$. This allows us to simplify and estimate the above inequality as

$$
N \geq\left(\begin{array}{l}
k-1 \\
r-1
\end{array}\right) \geq k-1 \geq \frac{k}{2}
$$

In the remaining case $k>l$, we can exploit that the number of positions in each column decreases from the left to the right to conclude that $k-l \geq r$. In the subcase $r=2$, we obtain

$$
N \geq \frac{l-1}{l} k \geq \frac{k}{2}
$$

If conversely $r>2$, we have the inequalities $1<r-1<k$ as well as $l \geq r$ and $k-1 \geq l$. Hence

$$
N \geq \frac{l-r+1}{l} \frac{k(k-1)}{2} \geq(l-r+1) \frac{k-1}{l} \frac{k}{2} \geq \frac{k}{2} .
$$


We next prove Theorem 9.1 under the additional assumption that the unitary operators $U$ in Definition 1.1 form a representation of the outer symmetry group.

Lemma 9.3. Suppose that $\left(H,\langle. \mid\rangle,.\left(E_{x}\right)_{x \in M}, P\right)$ is a discrete fermion system satisfying (9.1) and (9.2). Assume furthermore that there is a unitary representation of the outer symmetry group $\mathcal{O}$ on $H$ such that for every $\sigma \in \mathcal{O}$, the corresponding $U(\sigma)$ satisfies (1.2). Then the outer symmetry group cannot be the symmetric group $\mathcal{S}_{m}$.

Proof. Assume, on the contrary, that the fermion system has permutation symmetry $\mathcal{O}=\mathcal{S}_{m}$. Then, distinguishing the point $1 \in M$, the corresponding pinned symmetry group $\mathcal{R}$ is the group $\mathcal{S}_{m-1}$ of permutations of the other points $\{2, \ldots, M\}$. From (9.1), we know that $m>$ 3 , and thus we can for every $x \in M$ choose an even permutation $\sigma_{x} \in \mathcal{O}$ with $\sigma_{x}(1)=x$.

By assumption, $U$ is a representation of $\mathcal{S}_{m}$ on $H$. Let $V$ be the corresponding representation of $\mathcal{R}$ on $\hat{H}:=E_{1}(H)$ as given by (6.4). According to Lemma 2.10, the irreducible subspaces of $V$ can be chosen to be definite. Using Lemma 9.2 together with (9.1), one sees that $V$ must be the direct sum of trivial and sign representations. Since $\hat{H}$ has signature $(n, n)$, we can decompose it into a direct sum of the one-dimensional invariant subspaces

$$
\hat{H}=\bigoplus_{j=1}^{n} \hat{H}_{j}^{+} \oplus \bigoplus_{j=1}^{n} \hat{H}_{j}^{-},
$$

where the spaces $H_{j}^{+}$and $H_{j}^{-}$are positive and negative-definite, respectively.

Proposition 6.2 allows us to reconstruct $U$ from $V$. Let us consider what we get in the two cases when $V$ is the trivial or sign representation. For the trivial representation, we can assume that $\hat{H}=\mathbb{C}$. The construction of Proposition 6.2 yields $H=\mathbb{C}^{M}$ and

$$
E_{x}:\left(u_{x}\right)_{x \in M} \longmapsto\left(\delta_{x y} u_{x}\right)_{x \in M}, \quad U(\sigma):\left(u_{x}\right)_{x \in M} \longmapsto\left(u_{\sigma(x)}\right)_{x \in M} .
$$

In other words, $U$ is the standard representation of $\mathcal{O}$ on the complexvalued functions on $M$. The one-dimensional subspace spanned by the vector $(1, \ldots, 1) \in \mathbb{C}^{M}$ is clearly invariant; $U$ acts on it trivially. The orthogonal complement of this subspace is $(m-1)$-dimensional, and it is indeed irreducible, corresponding to the following Young diagram:

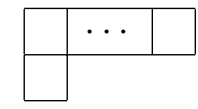


In view of (9.2), the fermionic projector must vanish identically on this $(m-1)$-dimensional irreducible subspace. We conclude that the subsystem corresponding to our one-dimensional representation of $V$ contains at most one particle.

In the case when $V$ is the sign representation, we can again assume that $\hat{H}=\mathbb{C}$. The construction of Proposition 6.2 yields the same discrete space-time as for the trivial representation, but now, using that the permutations $\sigma_{x}$ are all even,

$$
U(\sigma):\left(u_{x}\right)_{x \in M} \longmapsto\left(\operatorname{sgn}(\sigma) u_{\sigma(x)}\right)_{x \in M} .
$$

Since multiplying $U(\sigma)$ by the sign of $\sigma$ has no effect on whether a subspace in invariant, this representation has the same irreducible subspaces as the representation corresponding to a trivial $V$. Again, our subsystem contains at most one particle.

The uniqueness statement in Proposition 6.2 yields that $H$ is, in a suitable gauge, the direct sum of the scalar product spaces $\mathbb{C}^{m}$ obtained from each direct summand in (9.4). Since the spaces corresponding to the $H_{j}^{+}$are positive-definite, they must not contain any particles. As we saw above, each of the spaces corresponding to the $H_{j}^{-}$may contain at most one particle. Hence the total number of particles is at most $n$, contradicting (9.2).

The remaining task is to show that under the assumptions of Theorem 9.1, there is a representation $U$ of the outer symmetry group. Our strategy is to fix the discrete phase freedom completely, using special properties of the symmetric group. Then the resulting mapping $\sigma \mapsto U(\sigma)$ will be a unitary representation of $\mathcal{S}_{m}$. The next proposition gives us a group representation once the operators $U(\tau)$ are fixed up to a sign and are compatible with the group operations modulo signs. We denote the transposition of two points $x, y \in M, x \neq y$, by $\tau_{x, y}$. We let $\mathcal{T} \subset \mathcal{S}_{m}$ be the set of all transpositions. For the commutator of two group elements $g, h \in \mathcal{S}_{m}$ and two unitary operators $U_{1}, U_{2} \in U(H)$, we use the standard notations

$$
[g, h]:=g h g^{-1} h^{-1}, \quad\left[U_{1}, U_{2}\right]:=U_{1} U_{2} U_{1}^{-1} U_{2}^{-1} .
$$

Proposition 9.4. Let $U: \mathcal{T} \rightarrow U(H)$ be a mapping with the following properties:

(A) $U(\tau)^{2}=\mathbb{1}$ for all $\tau \in \mathcal{T}$.

(B) For all $\tau, \tau^{\prime} \in \mathcal{T}$ we have the implication

$$
\left[\tau, \tau^{\prime}\right]=\mathbb{1} \Longrightarrow\left[U(\tau), U\left(\tau^{\prime}\right)\right]=\mathbb{1} .
$$


(C) For all distinct $x, y, z \in M$,

$$
U\left(\tau_{x, y}\right) U\left(\tau_{y, z}\right) U\left(\tau_{x, y}\right)= \pm U\left(\tau_{x, z}\right) .
$$

Then there is a group representation $\hat{U}$ of $\mathcal{S}_{m}$ on $H$ with $\hat{U}(\tau)= \pm U(\tau)$.

Proof. Using the abbreviations $U_{x, y} \equiv U\left(\tau_{x, y}\right)$ and $\hat{U}_{x, y} \equiv \hat{U}\left(\tau_{x, y}\right)$, we define $\hat{U}_{1,2}$ by $\hat{U}_{1,2}=U_{1,2}$. The other operators are then introduced by conjugation, i.e., for all $x, y \in\{3, \ldots, m\}$,

$$
\begin{aligned}
\hat{U}_{1, y} & :=U_{2, y} \hat{U}_{1,2} U_{2, y}, \\
\hat{U}_{x, 2} & :=U_{1, x} \hat{U}_{1,2} U_{1, x}, \\
\hat{U}_{x, y} & :=U_{1, x} U_{2, y} \hat{U}_{1,2} U_{2, y} U_{1, x} .
\end{aligned}
$$

Note that the definition of $\hat{U}_{12}$ involves an arbitrariness of sign, because we are free to replace $U_{12}$ by $-U_{12}$. However, once the sign of $\hat{U}_{12}$ is fixed, the signs in (9.7)-(9.9) are determined, because the factors $U_{1, x}$ and $U_{2, y}$ always appear in pairs. A short calculation yields that $\hat{U}(\tau)= \pm U(\tau)$ and that the definition (9.9) is symmetric in $x$ and $y$. This implies that (A) and (B) remain valid if $U$ is replaced by $\hat{U}$. A direct calculation shows that in (C) the sign is now determined,

$$
\hat{U}_{x, y} \hat{U}_{y, z} \hat{U}_{x, y}=\hat{U}_{x, z} \text { for all distinct } x, y, z \in M .
$$

A general group element $g \in \mathcal{S}_{m}$ can be written as a product of transpositions,

$$
g=\tau_{1} \cdots \tau_{p} \quad \text { with } \tau_{i} \in \mathcal{T} .
$$

We claim that the corresponding $\hat{U}(g)$ is uniquely defined by

$$
\hat{U}(g)=\hat{U}\left(\tau_{1}\right) \cdots \hat{U}\left(\tau_{p}\right) .
$$

Indeed, if we represent $g$ in two different ways as products of transpositions, an elementary consideration shows that, using the rules (A), (B) and (9.10), we can iteratively transform the corresponding products (9.12) into each other. From (9.11) and (9.12), it immediately follows that $\hat{U}(g) \hat{U}(h)=$ $\hat{U}(g h)$, and thus $\hat{U}$ is the desired group representation of $\mathcal{S}_{m}$.

Before we can apply this proposition, we need to analyze the structure of a discrete fermion system with permutation symmetry in more detail. In view of the decomposition of Theorem 4.5, it suffices to consider a simple system. 
Lemma 9.5. Assume that $\left(H,\langle. \mid\rangle,.\left(E_{x}\right)_{x \in M}, P\right)$ is a simple system with outer symmetry group $\mathcal{O}=\mathcal{S}_{m}$. Assume furthermore that

$$
f<m \text { and } m>2 n \text {. }
$$

Then the system can be decomposed into a direct sum of simple subsystems

$$
H=\bigoplus_{k=1}^{K} H^{(k)}, \quad E_{x}=\bigoplus_{k=1}^{K} E_{x}^{(k)}, \quad P=\bigoplus_{k=1}^{K} P^{(k)}
$$

with $K \leq \min (2, n)$. The unitary operator $U$ in (1.2) can be chosen of the form

$$
U=F \cdot W(\sigma) \cdot \bigoplus_{k=1}^{K} U_{k}(\sigma)
$$

with arbitrary $F \in U(1)^{K}$. The mapping $U_{k}$ is defined only up to a discrete phase

$$
U_{k}: \mathcal{S}_{m} \longrightarrow U\left(H^{(k)}\right) / \mathbb{Z}_{f_{\text {sub }}} \text { with } f_{\text {sub }}=\operatorname{rank} P^{(k)} \geq 1
$$

The operator $W$ is trivial in the case $K=1$, whereas in the case $K=2$ it is the sign operator

$$
W: \mathcal{S}_{m} \longrightarrow \mathcal{S}_{2}: g \longmapsto \operatorname{sgn}(g)
$$

(where \pm 1 denote the neutral element and the transposition in $\mathcal{S}_{2}$, respectively).

Proof. Applying Proposition 4.7, Theorem 2.11 and Theorem 3.5, we obtain a decomposition of the form (9.14) and (9.15) with $K \in \mathbb{N}$. According to constructions (4.7) and (4.8), the direct summands are the simple systems, which all involve the same number of particles $f_{\text {sub }} \geq 1$. Furthermore, we know that the permutation operators $W$ form a homomorphism from $\mathcal{S}_{m}$ to $\mathcal{S}_{K}$ which acts transitively on $\{1, \ldots, K\}$.

Let us derive the inequality $K \leq n$ : We introduce for every $k \in\{1, \ldots, K\}$ the set

$$
M_{k}=\left\{x \in M \mid \operatorname{Tr}\left(E_{x}^{(k)} P^{(k)}\right)>0\right\} \subset M .
$$

From the completeness of the spectral projectors, we know that $\sum_{x \in M} \operatorname{Tr}$ $\left(E_{x}^{(k)} P^{(k)}\right)=\operatorname{Tr}\left(P^{(k)}\right)=f_{\text {sub }}$, and thus none of the sets $M_{k}$ is empty. We set $l=\# M_{1} \geq 1$. Since our system has the outer symmetry group $\mathcal{S}_{m}$, the set obtained from $M_{1}$ by a permutation of space-time points must be one of the other sets $M_{k}$. This gives rise to the lower bound

$$
K \geq\left(\begin{array}{c}
m \\
l
\end{array}\right) \text {. }
$$

This is consistent with the upper bound for the total number of particles in (9.13) only if $l=m$. Repeating this argument with $M_{1}$ replaced by any 
other $M_{k}$, we conclude that

$$
\operatorname{Tr}\left(E_{x}^{(k)} P^{(k)}\right)>0 \quad \text { for all } x \in M \text { and } k \in\{1, \ldots, K\} .
$$

In particular, the spin dimension $\left(p_{x}^{(k)}, q_{x}^{(k)}\right)$ of $E_{x}^{(k)}$ satisfies the condition $q_{x}^{(k)} \geq 1$ (because if $q_{x}^{(k)}$ were zero, the operator $E_{x}^{(k)}$ would project on a positive-definite subspace, and the local trace $\operatorname{Tr}\left(E_{x}^{(k)} P^{(k)}\right)$ would be negative). Using the direct sum structure (9.14), we obtain the desired inequality $K \leq n$.

By permuting the components, the operators $W(\sigma)$ have a natural action on $\mathbb{C}^{K}$, which makes the mapping $\sigma \mapsto W(\sigma)$ to a unitary representation of $\mathcal{S}_{m}$ on $\mathbb{C}^{K}$. Applying Lemma 9.2 together with the inequality $K \leq n$ and the second inequality in (9.13), we conclude that this representation decomposes into trivial and sign representations. In particular, for every even $\sigma$, the operator $W(\sigma)$ is the identity. As a consequence, for every odd permutation, $W(\sigma)$ transposes pairs of elements of the set $\{1, \ldots, K\}$. From the fact that for any odd $\sigma, \sigma^{\prime} \in \mathcal{S}_{m}$, the product $W(\sigma) W\left(\sigma^{\prime}\right)=W\left(\sigma \sigma^{\prime}\right)$ equals the identity, we deduce that $W(\sigma)$ is the same for all odd $\sigma$. The transitivity of $W$ implies that either $K=1$ and $W$ is trivial, or else $K=2$ and $W$ is the sign function (9.16).

Proof of Theorem 9.1. Assume that there is a discrete fermion system with permutation symmetry which satisfies the conditions (9.1) and (9.2). We decompose the system according to Theorem 4.5 into a direct sum of a trivial system and simple systems. Our goal is to construct a unitary representation of the outer symmetry group for each simple system. By taking the direct sum of these representations, we then obtain a representation for the whole discrete fermion system. This allows us to apply Lemma 9.3, giving a contradiction.

We thus consider a simple system, which for ease in notation we again denote by $\left(H,\langle. \mid\rangle,.\left(E_{x}\right)_{x \in M}, P\right)$. Representing the simple system as in Lemma 9.5 , we distinguish the cases $K=1$ and $K=2$. Furthermore, we shall treat the case $n=1$ separately, giving rise to the following three cases.

First case: $K=1$ and $n>1$. The operator $U(\tau)$ corresponding to any transposition $\tau \in \mathcal{T}$ is unique up to a phase factor (at this point it is more convenient not to impose the condition $\operatorname{det} U=1$, so that we have a continuous phase freedom). According to Theorem 3.5, the operators $U$ are compatible with the group operations up to a phase in the sense that for all $\tau, \tau^{\prime} \in \mathcal{T}, U(\tau) U\left(\tau^{\prime}\right)=\mathrm{e}^{\mathrm{i} \varphi} U\left(\tau \tau^{\prime}\right)$ with $\varphi \in \mathbb{R}$. In particular, $U(\tau)^{2}$ is a multiple of the identity. Thus by choosing the phase of $U(\tau)$ appropriately, we can arrange that condition (A) in Proposition 9.4 is satisfied. This fixes 
the operators $U(\tau)$ up to a sign. It remains to show that also conditions (B) and (C) in Proposition 9.4 hold.

We already know that $(\mathrm{C})$ holds with a more general phase factor, i.e., for all distinct $x, y, z \in M$,

$$
U_{x, y} U_{y, z} U_{x, y} U_{x, z}=\mathrm{e}^{\mathrm{i}} \vartheta(x, y, z) \mathbb{1},
$$

whereas in the Proof of Lemma 9.4 we used the notation $U_{x, y} \equiv U\left(\tau_{x, y}\right)$. The sign of the phase factor depends on our arbitrary choice of the signs of the operators $U(\tau)$. But up to the sign, the factor $\mathrm{e}^{\mathrm{i} \vartheta(x, y, z)}$ is well defined. From the permutation symmetry, we conclude that it is a constant independent of the space-time points, i.e.,

$$
U_{x, y} U_{y, z} U_{x, y} U_{x, z}= \pm \mathrm{e}^{\mathrm{i} \vartheta} \mathbb{1} \text { for all distinct } x, y, z \in M .
$$

Multiplying from the right by $U_{x, z}$ and from the left by $U_{x, y} U_{y, z} U_{x, y}$, we get the same relation, but with the sign of $\vartheta$ flipped. We conclude that $\mathrm{e}^{\mathrm{i} \vartheta}= \pm 1$. This proves $(\mathrm{C})$.

For the proof of (B) we first note that, due to the permutation symmetry, the commutator is a constant independent of the space-time points, i.e., there is a constant $\vartheta \in \mathbb{R}$ such that

$$
\left[U(\tau), U\left(\tau^{\prime}\right)\right]=\mathrm{e}^{\mathrm{i} \vartheta} \mathbb{1} \quad \text { for all } \tau, \tau^{\prime} \in \mathcal{T} \text { with }\left[\tau, \tau^{\prime}\right]=\mathbb{1} .
$$

Taking the adjoint of the commutator merely corresponds to exchanging $\tau$ and $\tau^{\prime}$. Hence the factor $\mathrm{e}^{\mathrm{i} \vartheta}$ is real.

It remains to rule out the case $\left[U(\tau), U\left(\tau^{\prime}\right)\right]=-\mathbb{1}$. We let $\tau_{i} \in \mathcal{S}_{m}, 1 \leq$ $i \leq p:=[(m-1) / 2]$, be the transposition of the space-time points $2 i-1$ and $2 i$. Then the transpositions $\tau_{1}, \ldots, \tau_{p}$ mutually commute. Moreover, the corresponding operators $U\left(\tau_{i}\right)$ map $\tilde{H}:=E_{m}(H)$ to itself. Denoting the restrictions of these operators to $\tilde{H}$ by $\tilde{U}\left(\tau_{i}\right)$, the relations $\tilde{U}\left(\tau_{i}\right)^{2}=\mathbb{1}$ and $\left[\tilde{U}(\tau), \tilde{U}\left(\tau^{\prime}\right)\right]=-\mathbb{1}$ give rise to the anti-commutation relations of a Clifford algebra,

$$
\tilde{U}\left(\tau_{i}\right) \tilde{U}\left(\tau_{j}\right)+\tilde{U}\left(\tau_{j}\right) \tilde{U}\left(\tau_{i}\right)=2 \delta_{i j} \mathbb{1}_{\tilde{H}}
$$

Considering the corresponding $c$-unitary group, we know from Lemma 2.10 that the Clifford representation splits into definite invariant subspaces. The irreducible Clifford representations are known explicitly (see, e.g. [5, Chapter I, Section 5]); they have dimension at least $2^{[p / 2]}$. We conclude that $n \geq 2^{[p / 2]}=2^{[(m-1) / 4]}$, in contradiction to $(9.1)$.

Second case: $K=2$. As in the first case, we consider the mutually commuting transpositions $\tau_{1}, \ldots, \tau_{p}$. Choosing corresponding unitary operators $U\left(\tau_{i}\right)$ satisfying (1.2), these operators map the subspace $\tilde{H}:=E_{m}(H)$ 
to itself; again we denote the restrictions to $\tilde{H}$ by $\tilde{U}\left(\tau_{i}\right)$. According to Theorem 3.5, $U\left(\tau_{i}\right)$ are compatible with the group operations in the sense that $U\left(\tau_{i}\right) U\left(\tau_{j}\right)=U\left(\tau_{i} \tau_{j}\right)$ modulo free gauge transformations in $U(1) \times$ $U(1)$. In particular, $U\left(\tau_{1}\right)^{2} \in U(1) \times U(1)$. As a consequence, using a block matrix notation in the index $k \in\{1,2\}$, the restriction of $U\left(\tau_{1}\right)$ to $\tilde{H}$ can be written as

$$
\tilde{U}\left(\tau_{1}\right)=\left(\begin{array}{cc}
0 & \mathrm{e}^{\mathrm{i} \beta} V^{-1} \\
\mathrm{e}^{\mathrm{i} \alpha} V & 0
\end{array}\right) \quad \text { with } \alpha, \beta \in[0,2 \pi),
$$

where $V$ is a unitary mapping from $\tilde{H}^{(1)}$ to $\tilde{H}^{(2)}$ with $\tilde{H}^{(k)}:=E_{m}^{(k)}\left(H^{(k)}\right)$. In order to satisfy condition (A), we need to chose $\beta=-\alpha$; this leaves us with one free parameter $\alpha$. Representing the operators $\tilde{U}\left(\tau_{2}\right), \ldots, \tilde{U}\left(\tau_{p}\right)$ similarly, we obtain the representations

$$
\tilde{U}\left(\tau_{i}\right)=\left(\begin{array}{cc}
0 & \mathrm{e}^{-\mathrm{i} \alpha_{i}} V_{i}^{-1} \\
\mathrm{e}^{\mathrm{i} \alpha_{i}} V_{i} & 0
\end{array}\right) \quad \text { with } \alpha_{i} \in[0,2 \pi),
$$

and unitary mappings $V_{i}: \tilde{H}^{(1)} \rightarrow \tilde{H}^{(2)}$.

We next consider for any distinct $i, j \in\{1, \ldots, p\}$, the commutator $\left[U\left(\tau_{i}\right)\right.$, $\left.U\left(\tau_{j}\right)\right]$ as defined by $(9.6)$,

$$
\left[U\left(\tau_{i}\right), U\left(\tau_{j}\right)\right]=\left(U\left(\tau_{i}\right) U\left(\tau_{j}\right)\right)^{2}
$$

Since $\tau_{i}$ and $\tau_{j}$ commute, this commutator must be an element of $U(1) \times U(1)$. Restricting to $\tilde{H}$ and using the representation (9.19), one sees that by choosing $\alpha_{j}$ appropriately, we can arrange that the commutator $\left[\tilde{U}\left(\tau_{i}\right), \tilde{U}\left(\tau_{j}\right)\right]$, and thus also the unrestricted commutator $\left[U\left(\tau_{i}\right), U\left(\tau_{j}\right)\right]$, is the identity. We choose the parameters $\alpha_{2}, \ldots, \alpha_{p}$ such that

$$
\left[U\left(\tau_{1}\right), U\left(\tau_{j}\right)\right]=\mathbb{1} \quad \forall j \in\{2, \ldots, p\} .
$$

This uniquely determines the operators $U\left(\tau_{2}\right), \ldots, U\left(\tau_{p}\right)$ up to signs. The only remaining free parameter $\alpha_{1}$ is of no relevance because the phase factors $\mathrm{e}^{ \pm \mathrm{i} \alpha_{1}}$ will drop out of all the following composite expressions.

Since all free parameters have been determined up to signs, we can use the permutation symmetry to conclude that the commutator (9.20) must be the same for all choices of $i, j \in\{2, \ldots, p\}$ (note that here we cannot choose $i=1$ or $j=1$ because $\tau_{1}$ is distinguished in (9.21)). In particular, since taking the adjoint of (9.20) corresponds to exchanging $i$ and $j$, we see 
that (9.20) is Hermitian. Thus there are the four possible cases

$$
\left(U\left(\tau_{i}\right), U\left(\tau_{j}\right)\right)^{2}=\left(\begin{array}{cc} 
\pm \mathbb{1}_{H^{(1)}} & 0 \\
0 & \pm \mathbb{1}_{H^{(2)}}
\end{array}\right)
$$

with arbitrary distributions of the signs. Multiplying (9.22) from the right by $U\left(\tau_{j}\right)$ and from the left by $U\left(\tau_{i}\right) U\left(\tau_{j}\right) U\left(\tau_{i}\right)$, the diagonal matrix on the right anti-commutes with $U\left(\tau_{j}\right)$ in view of (9.19). We thus obtain precisely (9.22), but with the two diagonal entries on the right exchanged. This rules out the two cases where the signs in (9.22) are opposite. In the case $\left[U\left(\tau_{i}\right), U\left(\tau_{j}\right)\right]=-\mathbb{1}$, the operators $\tilde{U}\left(\tau_{2}\right), \ldots, \tilde{U}\left(\tau_{p}\right)$ would satisfy (9.18), giving rise to a Clifford algebra with $p-1$ generators. Using that irreducible representations of this algebra have dimension at least $2^{[(p-1) / 2]}$ (see again [5]), we obtain a contradiction to (9.1).

We conclude that the operators $U\left(\tau_{i}\right)$ mutually commute. Permuting the space-time points $\{3, \ldots, m\}$ and repeating the above construction, one can arrange that $U\left(\tau_{1}\right)$ commutes with all $U(\tau)$ for which $\left[\tau_{1}, \tau\right]=0$. By subsequently commuting the points 1 and 2 with other space-time points and again repeating the above construction, we can arrange that (B) holds. We point out that the above construction has fixed the operators $U(\tau), \tau \in \mathcal{T}$, up to signs and up to the irrelevant phase parameter $\alpha_{1}$. The construction does not destroy the permutation symmetry in the sense that if we had started instead of $\tau_{1}$ with any other transposition, the resulting operators $U(\tau)$ would differ only by signs, and the parameter $\alpha_{1}$ may be different.

It remains to prove $(\mathrm{C})$. Using the permutation symmetry, we conclude that, similar to (9.17), there are parameters $\vartheta_{1}, \vartheta_{2} \in \mathbb{R}$ such that for all distinct $x, y, z \in M$,

$$
U_{x, y} U_{y, z} U_{x, y} U_{x, z}= \pm\left(\begin{array}{cc}
\mathrm{e}^{\mathrm{i} \vartheta_{1}} \mathbb{1}_{H^{(1)}} & 0 \\
0 & \mathrm{e}^{\mathrm{i} \vartheta_{2}} \mathbb{1}_{H^{(2)}}
\end{array}\right) .
$$

Multiplying from the right by $U_{x, z}$ and from the left by $U_{x, y} U_{y, z} U_{x, y}$, we can anti-commute the diagonal matrix on the right with $U_{x, z}$. We thus obtain the same relation, but with the replacements $\vartheta_{1} \leftrightarrow-\vartheta_{2}$. Hence $\vartheta_{1}=-\vartheta_{2}$. Moreover, we choose distinct points $a, b \in M$ which are all different from $x, y, z$ and assume without loss of generality that $m$ is different from all these points. Then we know from (B) that $U_{a, b}$ commutes with all the factors in (9.23), and thus

$$
\left[U_{a b}, U_{x, y} U_{y, z} U_{x, y} U_{x, z}\right]=\mathbb{1} .
$$

Furthermore, $\tilde{H}$ is invariant under all the operators under consideration. Evaluating the commutator (9.24) on the subspace $\tilde{H}$, we see from (9.23) 
and (9.19) that $\vartheta_{1}=\vartheta_{2}$. We conclude that $\mathrm{e}^{\mathrm{i} \vartheta_{1}}=1=\mathrm{e}^{\mathrm{i} \vartheta_{2}}$, and thus condition (C) is satisfied.

Third case: $K=1=n$. We remark that if $m>6$, we could proceed exactly as in the first case. The point of the following argument is that it applies also if the number of space-time points lies in the range $4 \leq m \leq 6$. Conditions (A) and (C) can be proved as in the first case. Also, for the proof of (B) we obtain exactly as in the first case that

$$
\left[\tau, \tau^{\prime}\right]=\mathbb{1} \Longrightarrow\left[U(\tau), U\left(\tau^{\prime}\right)\right]= \pm \mathbb{1}
$$

It remains to rule out the case of the minus sign. Thus assume that $[U(\tau)$, $\left.U\left(\tau^{\prime}\right)\right]=-\mathbb{1}$ for all commuting $\tau, \tau^{\prime} \in \mathcal{T}$. The operator $U_{1,2}$ is invariant on the subspaces $E_{3}(H)$ and $E_{4}(H)$. Since $U_{1,2}^{2}=\mathbb{1}$, the spectrum of the operator $\left.U_{1,2}\right|_{E_{3}(H)}$ is a subset of $\{1,-1\}$ and, due to permutation symmetry, it coincides up to a sign with the spectrum of $\left.U_{1,2}\right|_{E_{4}(H)}$. Furthermore, considering $\left\{\mathbb{1}, U_{1,2}\right\}$ as a representation of $\mathcal{S}_{2}$, we know from Lemma 2.10 that these operators can be diagonalized with definite eigenvectors. Since the spin dimension is $(1,1)$, one eigenvector is positive-definite and the other negative-definite.

Let us show that the spectrum of $\left.U_{1,2}\right|_{E_{3}(H)}$ cannot consist of one point. If this were the case, this operator would be a multiple of the identity, and it would be equal to either $+\left.U_{1,2}\right|_{E_{4}(H)}$ or $-\left.U_{1,2}\right|_{E_{4}(H)}$. In the first case, the restriction of $U_{1,2}$ to $E_{3}(H) \oplus E_{4}(H)$ would be a multiple of the identity, and would thus necessarily commute with $U_{3,4}$, a contradiction. In the second case, the symmetry condition $U_{12} P U_{12}=P$ would imply that $E_{3} P E_{4}=0$. The permutation symmetry would imply that $E_{x} P E_{y}=0$ for all $x \neq y$, and so $P$ would be invariant on all the subspaces $E_{x} P, x \in M$. Each of these subspaces would contain at least one particle, in contradiction to the upper bound in (9.2).

We just showed that the operator $\left.U_{1,2}\right|_{E_{3}(H)}$, and similarly $\left.U_{1,2}\right|_{E_{4}(H)}$, has the two eigenvalues +1 and -1 . We denote the corresponding orthogonal eigenvectors by $v_{3 / 4}^{ \pm}$, where we use the convention that the vectors $v_{x}^{+}$ and $v_{x}^{-}$are positive- and negative-definite, respectively. We next rule out the case that the vectors $v_{3}^{+}$and $v_{4}^{+}$correspond to the same eigenvalue: The operator $U_{3,4}$ anti-commutes with $U_{1,2}$, and furthermore it maps $E_{3}(H)$ into $E_{4}(H)$ and vice versa. This means that $U_{3,4}$ maps the eigenspaces of $E_{3}(H)$ to the eigenspaces of $E_{4}(H)$ corresponding to opposite eigenvalues. In particular, the positive-definite vector $v_{3}^{+}$is mapped to the negativedefinite vector $v_{4}^{-}$, in contradiction to the unitarity of $U_{3,4}$. 
Using (A) and (C) together with Proposition 9.4, we can arrange possibly by flipping signs that the operators $\left\{U_{1,2}, U_{2,3}\right\}$ generate a representation of $\mathcal{S}_{3}$ on $E_{4}(H)$. Completely reducing this representation into definite invariant subspaces, one sees that these invariant subspaces are spanned precisely by $v_{4}^{+}$and $v_{4}^{-}$. In other words, the two operators $U_{1,2}$ and $U_{2,3}$ have joint eigenvectors $v_{4}^{ \pm}$. Using the permutation symmetry, we can, at any point $x \in M$, choose two vectors $v_{x}^{+}$and $v_{x}^{-}$, the first being positive-definite and the second negative-definite. The operators $U_{x, y}$ map these vectors at the corresponding points into multiples each other, where positive- and negative-definite vectors are mapped to positive- and negative-definite vectors, respectively.

Using the symmetry condition $U_{1,2} P U_{1,2}=P$ together with our above observation that $v_{4}^{+}$and $v_{3}^{-}$lie in the same eigenspace of $U_{1,2}$, we conclude that the vector $E_{4} P v_{4}^{+}$is a multiple of $v_{4}^{+}$, whereas $E_{3} P v_{4}^{+}$is a multiple of $v_{3}^{-}$. More generally, using the permutation symmetry, we get

$$
\begin{aligned}
& E_{4} P v_{4}^{+}=\lambda v_{4}^{+} \quad \text { and } \quad E_{x} P v_{4}^{+}=c v_{x}^{-}, \quad x \neq 4 \\
& E_{3} P v_{3}^{+}=\lambda v_{3}^{+} \quad \text { and } \quad E_{x} P v_{3}^{+}=d v_{x}^{-}, \quad x \neq 3
\end{aligned}
$$

with coefficients $\lambda, c, d \in \mathbb{C}$. Here we used the permutation symmetry to arrange that the coefficients $c$ and $d$ are independent of $x$. Using (1.1) and the fact that $P$ is a projector, it follows that

$$
0=\left\langle v_{3}^{+} \mid P v_{4}^{+}\right\rangle=\left\langle P v_{3}^{+} \mid P v_{4}^{+}\right\rangle=\bar{d} c \sum_{x \neq 3,4}\left\langle v_{x}^{-} \mid v_{x}^{-}\right\rangle .
$$

We conclude that $c=0$ or $d=0$. If, e.g., $c=0$, it follows that $P v_{4}^{+}=\lambda v_{4}^{+}$, and the permutation symmetry yields that even $P v_{x}^{+}=\lambda v_{x}^{+}$for all $x \in M$. If $\lambda \neq 0$, the vectors $v_{x}^{+}$would all lie in the image of $P$, in contradiction to (9.2). We conclude that $\lambda$ vanishes and thus $P v_{x}^{+}=0$ for all $x \in M$. Repeating the argument of this paragraph with all indices + and - reversed, we obtain similarly that $P v_{x}^{-}=0$ for all $x \in M$. Hence $P$ vanishes identically, in contradiction to $(9.2)$.

\section{Acknowledgments}

I thank Florian Brückl and the referee for valuable comments.

\section{References}

[1] F. Finster, The principle of the fermionic projector, AMS/IP Stud. Adv. Math. 35 (2006). 
[2] F. Finster, A variational principle in discrete space-time - existence of minimizers, Calc. Var. PDEs, Doi: 10.1007/s00526-006-0042-0 [math$\mathrm{ph} / 0503069]$.

[3] F. Finster, The principle of the fermionic projector: an approach for quantum gravity?, gr-qc/0601128, in "Quantum Gravity," B. Fauser, J. Tolksdorf and E. Zeidler, Eds., Birkhäuser Verlag (2006).

[4] L. Fuchs, Abelian groups, Pergamon Press, 1960.

[5] H.B. Lawson and M.-L. Michelsohn, Spin geometry, Princeton University Press, 1989.

[6] S. Sternberg, Group theory and physics, Cambridge University Press, 1994. 